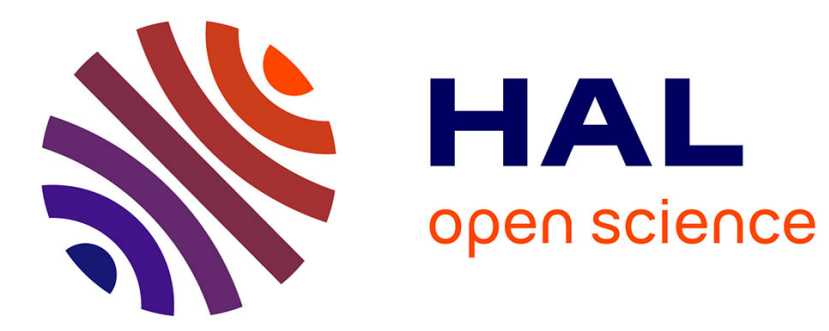

\title{
Public transport reliability and commuter strategy
}

Guillaume Monchambert, André de Palma

\section{To cite this version:}

Guillaume Monchambert, André de Palma. Public transport reliability and commuter strategy. Journal of Urban Economics, 2014, 81, pp.14-29. 10.1016/j.jue.2014.02.001 . hal-00827972v2

\section{HAL Id: hal-00827972 \\ https://hal.science/hal-00827972v2}

Submitted on 17 Apr 2015

HAL is a multi-disciplinary open access archive for the deposit and dissemination of scientific research documents, whether they are published or not. The documents may come from teaching and research institutions in France or abroad, or from public or private research centers.
L'archive ouverte pluridisciplinaire HAL, est destinée au dépôt et à la diffusion de documents scientifiques de niveau recherche, publiés ou non, émanant des établissements d'enseignement et de recherche français ou étrangers, des laboratoires publics ou privés. 


\title{
Public transport reliability and commuter strategy
}

\author{
Guillaume Monchambert ${ }^{\mathrm{a}, *}$, André de Palma ${ }^{\mathrm{a}, \mathrm{b}}$ \\ ${ }^{a}$ Ecole Normale Supérieure de Cachan \\ ${ }^{b}$ Ecole Polytechnique
}

\begin{abstract}
We consider the modeling of a bi-modal competitive network involving a public transport mode, which may be unreliable, and an alternative mode. Commuters select a transport mode and their arrival time at the station when they use public transport. The public transport reliability set by the public transport firm at the competitive equilibrium increases with the alternative mode fare, via a demand effect. This is reminiscent of the Mohring effect. The study of the optimal service quality shows that often, public transport reliability and thereby patronage are lower at equilibrium compared to first-best social optimum. The paper provides some public policy insights.
\end{abstract}

Keywords: public transport; reliability; duopoly; welfare; Mohring effect; schedule delay JEL: R41; R48; D43

\section{Introduction}

Despite increasing pollution and congestion in cities, cars remain the most popular mode of transport. Therefore, improving alternative modes of transport and making them attractive is essential in an urban context. Even if travel time is presented as the main determinant of trip characteristics, Beirao and Cabral (2007) have shown that increasing the service quality remains an important determinant of public transport demand. Several studies strongly suggest that reliability (understood as punctuality) of public transport is crucial to leverage the demand (Bates et al., 2001; Hensher et al., 2003; Paulley et al., 2006; Coulombel and de Palma, 2014), and in their qualitative review, Redman et al. (2013) claim that reliability is the most important quality attribute of public transport for users.

Although there is a long literature about road reliability, a sensitive lack of research is observed in public transport field (Bates et al., 2001). Some studies highlight a valuation of road reliability (Bates et al., 2001; Fosgerau and Karlström, 2010), others underline the importance of public transport comfort (de Palma et al., 2013) or punctuality (Jensen, 1999), but only few deal with reliability in analytical way. A meeting of two persons has been analyzed in the context of game theory by Fosgerau et al. (2014). Public transport imposes specifications that will be exploited here.

This paper focuses on the two-way implication between punctuality level of public transport and commuter behavior. On one hand, the transportation lack of punctuality plays an important role

${ }^{*}$ Corresponding Author: Guillaume Monchambert, Ecole Normale Supérieure de Cachan, Laplace 302, 61 avenue du Président Wilson, 94235 Cachan, France - Phone: +33147402316.

Email addresses: guillaume.monchambert@ens-cachan.fr (Guillaume Monchambert), andre.depalma@ens-cachan.fr (André de Palma)

January 30, 2013 
in the modal shift as commuters may incur extra-cost due to waiting time, arriving late or missing the bus. On the other hand, Mohring (1972) has pointed out that scheduled urban public transport is characterized by increasing returns to scale. According to the Mohring Effect, as transportation patronage increases, the operator tends to improve the frequency of service and to provide external benefits due to reduced waiting times and denser transit network. Demand is also influential in the service quality offered, and the bus company may adapt its punctuality to the level of potential demand. We show that some users may decide to arrive late at the bus stop when punctuality is low. As a consequence, the bus company itself may become less strict as far as the punctuality. In a nutshell, this means that user behavior (punctuality of users) is influenced by the punctuality of public transport. These mechanisms may generate a vicious circle: lateness of some agents calling for lateness of the other agents.

In this paper, we study three situations: (i) the reaction of the bus company when it faces a higher price of the alternative mode, (ii) the gap between the bus punctuality at equilibrium and at optimum and (iii) the equilibrium versus optimal modal split when punctuality matters. In particular, we show that when the alternative mode fare raises, the resulting increase in bus patronage makes the bus operator improve the bus punctuality. This mechanism can be considered as an application of the "Mohring Effect".

We consider a duopoly which symbolizes a modal competition between public transport and another mode, which we call taxi. The attention is focused on the monetary impacts of punctuality. We simplify aspects related to engineering. A duopoly is used because determinants of demand for public transport are related to the demand for private transport (Balcombe et al., 2004). Usually, the public transport firm is not profit maximizing because it is regulated and because it receives subsidies. Some intermediary cases arise when the firm receives fixed subsidies and wishes to maximize its revenues and minimize its costs. In order to guarantee good enough quality of service, the subsidy may depend on the quality of service offered. Such incentives are more generally used when public resources are scarce. Two different types of variables are observed in the model: the public transport punctuality level, which is selected by the bus company and the prices set by the bus and taxi companies. Both have a substantial influence on demand for public transport (Paulley et al., 2006). Unreliability has a strong negative impact as it implies excessive waiting time and uncertainty (Wardman, 2004; Paulley et al., 2006).

We insist on the fact that even if along the paper we consider the alternative mode as a taxi company, the analysis may easily be extended to the private car. In fact, one has to only consider the taxi fare as an exogenous variable which stand for the variable cost of using a car. An increase in taxi fare can also be interpreted as a rise in gas prices.

Considering commuting trips, preferences can be analyzed with the dynamic scheduling model. In this model, individuals' preferences reflect agents' tradeoff between travel time, early schedule and late schedule delays. Commuters may choose different strategies to minimize their trip cost. This theory has been first introduced by Vickrey (1969) and then renewed by Arnott et al. (1990). Such analysis is usually specific to road analysis (Fosgerau and Karlström, 2010); here we introduce waiting time to extend this model to public transport. Incidentally, note that the French State-owned railroad (SNCF) suggests to reschedule work arrival and departure times in order to reduce congestion (Steinmann, 


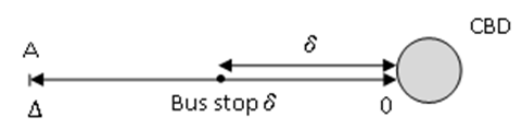

Figure 1: The route from home to CBD

2013).

Commuters are differentiated by their preferred arrival time at workplace and by their residential location, which is measured as the time to travel to their destination when using the alternative mode. Two different preferred arrival times are considered, and the location is uniformly distributed among commuters.

The analysis for the model proceeds in three steps. The first step consists in finding out the modal choice of commuters depending on prices and punctuality for the public transport and the alternative mode. The second step determines which price and punctuality levels are set by companies at equilibrium given the behavior of commuters identified in step one. The third step is to assess the prices and the punctuality level that minimize the total social cost and to compare these results with the ones derived in step two.

Our model is here applied to road modes, but it can be generalized to other transport modes which face delays, such as inter-city rail or air transport. More generally, the modeling approach is relevant for any service concerned with reliability.

The paper is organized as follows. Section 2 describes the model and the commuter's strategies. Section 3 considers equilibrium and its properties. The gain due the transition from equilibrium to optimum is analyzed in Section 4. A numerical application is provided in Section 5 to illustrate our results and to present some public policy insights. In Section 6, we propose an extension by assuming that a second bus arrives shortly after the first one. The final section concludes and provides directions for further research.

\section{Punctuality in public transport}

We consider an unique route from an origin A to a destination B with households living alongside this route. Every morning, all commuters have to reach the point B which can be viewed as the CBD of a city.The route is measured in time units and is $\Delta$ hours long. A unique bus line and a taxi company serve the CBD by using this route and bus stations are uniformly distributed alongside this route.

As both modes are road modes, they endure the same traffic conditions. Therefore, we do not take into account congestion on the road. Moreover, the road congestion has no impact on the modal split. Thus, both modes have the same speed, and we refer to a bus stop located at $\delta$ hours from the CBD as "bus stop $\delta "$. For example, the bus stop $\Delta$ is located at the border of the city. Similarly, all commuters live along the route, and we refer to commuters who need $\delta$ hours to reach the CBD, whether they use the bus service or the taxi service, as "commuters $\delta$ ". For each $\delta \in[0 ; \Delta]$, all commuters $\delta$ live at the same place (see Figure 1).

The commuters are divided into two groups according to their preferred arrival times: the first type of commuters (referred to as Group A, which includes a part $\theta$ of the population) would rather arrive at time $T$, and the second one (referred to as Group B) at time $T+x$ (see Figure 2). This reflects the 


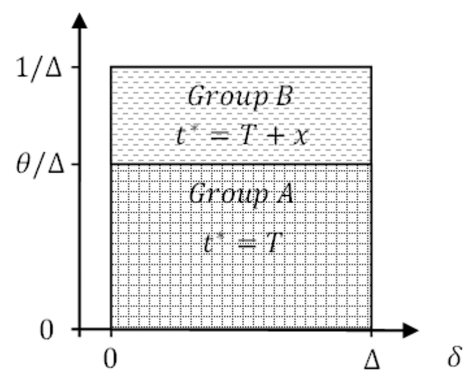

Figure 2: Distribution of taxi trip time in Group A and Group B

fact that even though a majority of commuters wishes to arrive at work place at the same time, not all commuters have the same preferred arrival time. Commuters locations are uniformly distributed among each group in the same manner (Figure 2) and the distribution is assumed to have a support $[0 ; \Delta]$ so that $F(0)=0$ and $F(\Delta)=1$.

For analytical tractability, we consider a single bus. However, this model can be easily adapted to other modes of public transport that run on a schedule. The bus is scheduled to arrive at the CBD at a given time, but it may be late. The probability of lateness is not random: the bus company selects its quality service level and applies it in the same manner along the route. Thus, when the bus company chooses to be late, it is late along the whole journey, and its lateness is constant over time. Commuters are aware of the punctuality level and adapt their behavior accordingly. In particular, they might arrive at the bus stop after the scheduled time even if there is a risk to miss the bus by doing so. This late arrival can occur rationally because there is a waiting cost for users. Commuters optimize their tradeoffs between cost of waiting time, schedule delay, and a cost corresponding to the use of alternative mode, which in our model is the taxi. A commuter may either select taxi ex ante or use the taxi if he misses the bus.

It should be stressed that the taxi service represents all private transport modes. From user perspective, the taxi fare is not different than the variable cost of his own car.

Table 1 introduces notations used in this paper and their numerical values that will be used in Section 2, 5 and 6.We first characterize the network and then the commuter behavior. Finally, we characterize the modal split.

\subsection{Transport supply}

Bus stops are uniformly distributed between 0 and $\Delta$. The bus is scheduled to arrive at its destination, the CBD, at time $T$. As there is no road congestion, it is also scheduled to serve the bus stop $\delta$ at time $T-\delta$ and to leave at time $T-\delta$ i.e there is no transfer cost. ${ }^{1}$ The bus company may choose that the bus is late and arrives at CBD time $T+x$. In this case, the bus stops at every bus stop $\delta$ at time $T+x-\delta$. The bus arrives at the CBD at time $T$ with probability $P$ and at time $T+x$ with probability $1-P$. Whatever the bus lateness, the total bus trip time is constant and equals to $\Delta$. The potential lateness is also constant and equals to $x$.

\footnotetext{
${ }^{1}$ The loading time is assumed to be set to zero without loss of generality.
} 


\begin{tabular}{ccc}
\hline Parameter & Comment & Suggested value \\
\hline$T$ & Scheduled arrival time & - \\
$x$ & Lateness & $10 / 60$ (hour) \\
$P \in\left[\frac{1}{2} ; 1\right]$ & Probability of the bus being on time & - \\
$t_{p} \in\{T ; T+x\}$ & Arrival time at the bus stop of the bus & - \\
$\delta \in[0 ; \Delta]$ & Taxi (or bus) trip time & (hour) \\
$\Delta$ & Maximal taxi trip time & $35 / 60$ (hour) \\
$t_{*}^{*} \in\{T ; T+x\}$ & Preferred arrival time of users & - \\
$t_{a} \in\{T ; T+x\}$ & Arrival time at the bus stop of the user & - \\
$\theta \in\left[\frac{1}{2} ; 1\right]$ & Share of population in Group $A$ & - \\
$\alpha_{b u s}$ & In-bus time cost per unit of time & $15(\$ /$ hour $)$ \\
$\alpha_{\text {taxi }}$ & In-taxi time cost per unit of time & $4(\$ /$ hour $)$ \\
$\eta$ & Waiting time cost per unit of time & $20(\$ /$ hour $)$ \\
$\gamma$ & Early delay cost per unit of time & $10(\$ /$ hour $)$ \\
$\kappa$ & Late delay cost per unit of time & $30(\$ /$ hour $)$ \\
$\tau$ & Bus fare & $(\$)$ \\
$c$ & Taxi fare & $(\$ /$ hour $)$ \\
$d$ & Operating cost per unit of time (taxi) & $40(\$ /$ hour $)$ \\
\hline
\end{tabular}

Table 1: Parameters values

The probability of the bus being on time is endogenous: the bus company sets its level. It does not depend on traffic conditions, number of passengers or loading time. The worst quality of service occurs when the bus has the same probability of being on time and being late. We assume that a regulator imposes this constraint to assure a consistent timetable ${ }^{2}$. The "punctuality level" corresponds to the probability of the bus being on time.

Assumption 1. The probability $P$ of the bus being on time satisfies the following inequality:

$$
\frac{1}{2} \leq P \leq 1
$$

We assume that there is no capacity constraint in the bus. The bus fare, priced by the bus company, is $\kappa$ for each passenger.

Commuters have an access to an alternative mode of transport. In our model we consider this option as a taxi service, but it can also be walking or personal car use. The taxi company sets a fare $\tau$ which corresponds to the price charged per minute of travel.

\footnotetext{
${ }^{2}$ Minimal value of $P$ is $1 / 2$, otherwise we would face another schedule than the expected one. Indeed, if $P$ is lower than $1 / 2$, then the bus is more often late than on time. Commuters may also consider that the bus becomes more punctual according to a new "unofficial" timetable where the bus is supposed to arrive at time $T+x$.
} 


\subsection{Demand for bus and taxi}

Commuters are assumed to incur a schedule delay cost if they arrive at time $t \neq t^{*}, t^{*}$ being their preferred arrival time. There is no transfer cost: commuters do not incur a cost by reaching the bus stop as the bus stops are assumed to be uniformly distributed along the route where the commuters live.

A commuter has a choice between catching the bus and using the taxi service. However, he may miss the bus, and then, he has to use the taxi service. We assume the headway is so long that all users who miss the bus prefer to use the taxi service ${ }^{3}$. If he tries to catch the bus, the commuter $\delta$ uses the bus stop $\delta$ because it minimizes his transfer cost. A commuter $\delta$ choosing to catch the bus bears the following schedule delay cost function that is assumed to depend on the bus arrival time at the station, denoted $t_{a} \in\{T-\delta ; T-\delta+x\}$, the arrival time of the bus at the bus station, denoted $t_{p} \in\{T-\delta ; T-\delta+x\}$, its most preferred trip time, denoted $t^{*} \in\{T ; T+x\}$ as well as on the arrival time at destination of the bus, denoted $t_{d} \in\{T ; T+x\}$ :

$$
C C_{\text {bus }}= \begin{cases}\kappa+\delta \alpha_{\text {bus }}+\eta\left(t_{p}-t_{a}\right)+\beta\left[t^{*}-t_{d}\right]^{+}+\gamma\left[t_{d}-t^{*}\right]^{+} & \text {if }\left(t_{a} \leq t_{p}\right), \\ \delta\left(\alpha_{\text {taxi }}+\tau\right)+\gamma\left[t_{d}-t^{*}\right]^{+} & \text {if }\left(t_{a}>t_{p}\right),\end{cases}
$$

with $[x]^{+}=x$ if $x \geq 0$ and 0 if $x<0, \kappa$ the bus fare, $\alpha_{\text {bus }}$ the in-bus time cost, $\eta$ the waiting time

cost, $\beta$ the early delay cost, $\gamma$ the late delay cost, $\alpha_{\text {taxi }}$ the in-taxi time cost, $\tau$ the taxi fare and $\delta$ the trip time of commuter $\delta$.

If a commuter chooses to use the taxi service from the start, he incurs the following cost:

$$
C C_{\text {taxi }}=\delta\left(\alpha_{\text {taxi }}+\tau\right)
$$

with $\alpha_{\text {taxi }}$ the taxi travel time value, $\tau$ the taxi fare and $\delta$ the taxi trip time.

By considering that for all $\delta \in[0 ; \Delta]$, the value of time in bus $\delta \alpha_{b u s}$ is incurred by commuter $\delta$ whatever is its choice, we can normalize the cost functions to:

$$
\begin{gathered}
C C_{b u s}= \begin{cases}\kappa+\eta\left(t_{p}-t_{a}\right)+\beta\left[t^{*}-t_{d}\right]^{+}+\gamma\left[t_{d}-t^{*}\right]^{+} & \text {if }\left(t_{a} \leq t_{p}\right), \\
\delta(\check{\alpha}+\tau)+\gamma\left[t_{d}-t^{*}\right]^{+} & \text {if }\left(t_{a}>t_{p}\right),\end{cases} \\
C C_{\text {taxi }}=\delta(\check{\alpha}+\tau),
\end{gathered}
$$

with $\check{\alpha}=\alpha_{\text {taxi }}-\alpha_{\text {bus }}$.

Assumption 2. The cost of waiting one minute for a bus, $\eta$, is lower than the cost of being one minute late, $\gamma$, and higher than the cost of being one minute early, $\beta$ :

$$
\gamma \geq \eta \geq \beta
$$

This assumption is consistent with literature valuations (Wardman, 2004).

\footnotetext{
${ }^{3}$ This restriction is removed in the extension presented in Section 6 .
} 


\subsection{Commuters' strategies}

Commuters dispose of three different strategies to minimize the cost of a trip. A strategy is defined by an arrival time at the bus stop. Arriving at the bus stop at time $T$ corresponds to Strategy $O$ (On-time at the bus stop), arriving at time $T+x$ to Strategy $L$ (Late at the bus stop) and Strategy $T$ (Taxi) embodies the decision to use the taxi. If a commuter chooses Strategy $O$, he waits until the bus arrives; if he chooses Strategy L, he uses the taxi service only in the case he misses the bus. Strategy T corresponds to the choice of the taxi cab at the beginning of the trip.

As a convention, we assume that a commuter who is indifferent between two strategies has a preference for maximizing its chance to get the bus. The commuter also chooses:

Strategy $O$ (arrive at time $T$ ) if $E C(O) \leq E C(T)$ and $E C(O) \leq E C(L)$;

Strategy $L$ (arrive at time $T+x$ ) if $E C(L)<E C(O)$ and $E C(L) \leq E C(T)$;

Strategy $T$ (choose the taxi) if $E C(T)<E C(O)$ and $E C(T)<E C(L)$,

where $E C(i)$ represents the expected cost of strategy $i$.

Proposition 1. Under Assumption 1 and Assumption 2, the commuter $\delta$ in Group A selects:

$$
\begin{gathered}
\text { Strategy } O \text { (time } T) \quad \text { if } \quad \delta \geq \delta_{T, O}^{A}, \\
\text { Strategy } T \text { (taxi) } \quad \text { if } \quad \delta<\delta_{T, O}^{A},
\end{gathered}
$$

where $\delta_{T, O}^{A} \equiv[\kappa+(1-P)(\eta+\gamma) x] /(\check{\alpha}+\tau)$.

Proof. See AppendixA.

For a commuter wishing to arrive at time $T$, Strategy $L$ is never selected. A commuter chooses Strategy $L$ instead of Strategy $T$ if he prefers a late bus trip over a taxi trip. However such a commuter prefers an on time bus trip over taxi trip and consequently, he will choose Strategy $O$.

Proposition 2. Under Assumption 1 and Assumption 2, the commuter $\delta$ in Group B selects:

$$
\begin{array}{ccc}
\text { Strategy } O \text { (time } T) & \text { if } & \delta \geq \delta_{L, O}^{B}, \\
\text { Strategy } L(\text { time } T+x) & \text { if } & \delta_{T, L}^{B} \leq \delta<\delta_{L, O}^{B}, \\
\text { Strategy } T \text { (taxi) } & \text { if } & \delta<\delta_{T, L}^{B},
\end{array}
$$

where $\delta_{L, O}^{B} \equiv\left[\kappa+\left(\frac{1-P}{P} \eta+\beta\right) x\right] /(\check{\alpha}+\tau)$ and $\delta_{T, L}^{B} \equiv \kappa /(\check{\alpha}+\tau)$.

Proof. See AppendixB.

Strategy $L$ is selected by some commuters from Group B unlike commuters from Group A. It can be explained by the fact that for Group B, Strategy $L$ corresponds to a possibility of the bus arriving on time without extra waiting time. A commuter who prefers an on-time bus trip over a taxi trip, and prefers a taxi trip over an early-arrival bus trip, chooses Strategy $L$.

The share of commuters choosing Strategy $T$ is independent of the probability of the bus being on time. $P$ has no influence in the arbitrage between Strategy $L$ and Strategy $T$. For commuters in Group B, choosing Strategy $L$ is equivalent to choosing Strategy $T$ except that they take the bus when it is late. Consequently, Strategy $L$ is preferred to Strategy $T$ as long as the cost of taking the 


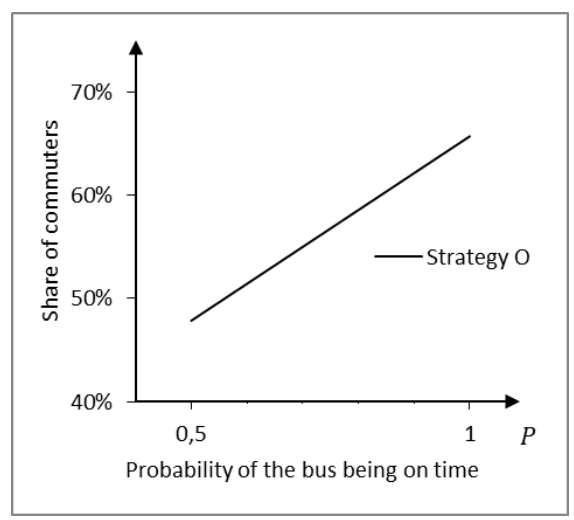

Group A

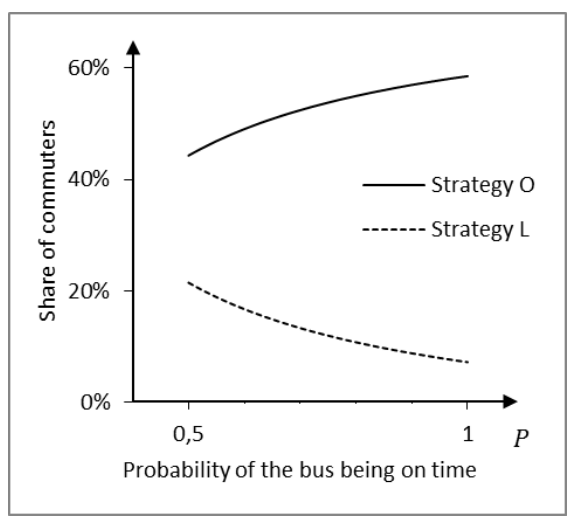

Group B

Figure 3: Share of commuter choosing Strategy $O$ and Strategy $L$ as a function of $P$, the probability of the bus being on time $(\kappa=8, \tau=50)$

bus when it is late is lower than the cost of taking a taxi. Then, this arbitrage is independent of the probability of the bus being on time.

When the punctuality decreases, the share of commuters arriving late at the bus stop increases. The cut in the service quality makes the cost of Strategy $O$ higher (because of A.2) and the cost of Strategy $L$ smaller (except for commuters living so close to the CBD that a taxi trip is still cheaper than a bus trip, but we do not take account of these commuters because they still prefer Strategy T). Then, among the commuters who chose Strategy $O$ before the service quality falls, those living the closest to the CBD are the most indifferent between both strategies, and switch from Strategy $O$ to Strategy L. Moreover, the bus company itself may become less strict, and generate a vicious circle.

When the taxi fare, $\tau$, increases, more commuters choose to arrive at the bus stop at $T$, and less commuters choose Strategy $L$ and Strategy $T$. This is due to the fact that on the one hand some commuters have a bigger interest to minimize the probability of taking the taxi by shifting from Strategy $L$ to Strategy $O$ and from Strategy $T$ to Strategy L. On the other hand, the shift from Strategy $L$ to Strategy $O$ is larger than the one from Strategy $T$ to Strategy $L$.

Figure 3 illustrates these results. Other things being equal, the share of commuters arriving at $T$ (and by doing so they are sure to catch the bus) among Group $A$ increases from around $48 \%$ when $P=1 / 2$ to almost $65 \%$ when $P=1$. The share of commuters in Group $B$ choosing to arrive late at the bus stop (Strategy $L$ ) depends inversely on the probability of the bus being on time. If the bus arrives later, some users switch from Strategy $O$ to Strategy $L$ which leaves the bus company no incentive to restore the service quality.

Assumption 3. The maximum cost of the taxi use, priced at the operating cost, is higher than the cost of the bus use, when priced at zero and when the bus arrives on time with probability $1 / 2$ :

$$
\Delta(\check{\alpha}+d) \geq \frac{1}{2}(\eta+\gamma) x .
$$

Once the commuters' strategies are defined, shares of commuters who are at the bus stop at time 
$T$ or $T+x$ are known. Demands are described by

$$
\begin{aligned}
D_{\text {bus }} & =\theta\left(1-\frac{\delta_{T, O}^{A}}{\Delta}\right)+(1-\theta)\left[1-\frac{\delta_{L, O}^{B}}{\Delta}+(1-P) \frac{\delta_{L, O}^{B}-\delta_{T, L}^{B}}{\Delta}\right], \\
D_{\text {taxi }} & =\theta \frac{\delta_{T, O}^{A}}{\Delta}+(1-\theta)\left[P \frac{\delta_{L, O}^{B}-\delta_{T, L}^{B}}{\Delta}+\frac{\delta_{T, L}^{B}}{\Delta}\right] .
\end{aligned}
$$

Thus, the bus (and taxi) patronage depends on the probability of the bus being on time ${ }^{4}$. Group $A$ is more sensitive to the service quality than $\operatorname{Group} B$ (see also Figure 3 ). This is due to the fact that commuters from Group A incur late arrival costs while commuters from Group B incur early arrival costs and, as seen in Assumption 2, the penalty for lateness is much higher than the penalty for arriving early at the destination.

\section{Competition between bus and taxi companies}

In this section, we explore equilibrium pricing and punctuality level in a duopoly competition. We assume that following condition holds:

$$
\Delta(\check{\alpha}+\tau) \geq \kappa+\frac{1}{2}(\eta+\gamma) x .
$$

ition assures an interior solution.We will check if it holds once the equilibrium values of $\tau$ and $\kappa$ are solved.

Some situations where one mode takes over the whole patronage are worth considering. They are characterized by corner solutions and Condition (4) does not hold. We identify three potentials elements that may cause such situations. First, if the bus fare is too high, even commuters living the farthest from the center choose the taxi. Second, commuters use the taxi service when the potential late arrival of the bus is too costly. This may be due to a very large delay or to a very high valuation of scheduling costs by commuters. If commuters attach importance to be on time without wasting time, then they give priority to the most punctual mode, the taxi service. Finally, when the cost associated to the taxi travel with respect to the bus journey is too high, no one will use the taxi service. These three arguments might help to explain why, in some cities, one specific transport mode is predominant.

Both companies incur a cost. The cost incurred by the bus company only depends on the punctuality level and is assumed to be quadratic. It is a sunk cost in the sense of being unrecoverable (Sutton, 1991). The cost of the taxi company linearly depends on the total travel time and can be viewed as an operating cost:

$$
\begin{gathered}
\text { Cost }_{\text {bus }}=\frac{c}{2} P^{2}, \\
\text { Cost }_{\text {taxi }}=d * T T T,
\end{gathered}
$$

with $c$ the punctuality cost, $d$ being the cost per hour traveled, and TTT the total travel time of the taxi company. Note that the bus company cost does not depend on the travel time. As the travel time

\footnotetext{
${ }^{4}$ We assume perfect information concerning the service quality. Otherwise, the service would be an experience based good involving learning and the user's level of risk aversion should be taken into account.
} 
is constant, the linked cost does not matter in the objectification process. The punctuality is a costly component because being punctual implies on the one hand a high pressure on bus drivers to make them arrive on time, and on the other the set-up of an efficient organization to pick up and drop off the commuters.

The bus company chooses the bus fare $\kappa$ and the punctuality level $P$, so as to maximize its profit like a classical firm. From equations (3a) and (5a), the bus company profit can be written as

$$
\Pi_{b u s}=\kappa D_{b u s}-\frac{c}{2} P^{2} .
$$

There exists a unique solution ${ }^{5}$ satisfying the first-order conditions $\partial \Pi_{b u s} / \partial \kappa=0$ and $\partial \Pi_{b u s} / \partial P=$ 0 , given by

$$
\begin{aligned}
& \kappa^{e}= \frac{1}{2}\left(\check{\triangle}^{e}-\Gamma^{e} x\right), \\
& P^{e}= \begin{cases}\frac{1}{2} & \text { if } c>c_{2}^{e}, \\
\frac{\kappa^{e} \breve{\eta} x}{c \check{\triangle}^{e}} & \text { if } c \in\left[c_{1}^{e} ; c_{2}^{e}\right], \\
1 & \text { if } c<c_{1}^{e},\end{cases}
\end{aligned}
$$

where $\check{\Delta}^{e}=\Delta\left(\check{\alpha}+\tau^{e}\right), \Gamma^{e}=\left(1-P^{e}\right) \eta+(1-\theta) P^{e} \beta+\theta\left(1-P^{e}\right) \gamma, \check{\eta}=\eta-(1-\theta) \beta+\theta \gamma, c_{1}^{e} \equiv$ $\kappa^{e} \check{\eta} x / \breve{\Delta}^{e}$ and where $c_{2}^{e} \equiv 2 c_{1}^{e}$.

The price of an hour traveled in a taxi, $\tau$, is set by the taxi company to maximize its profit. From equations (3b) and (5b), taxi profit is given by

$$
\begin{aligned}
\Pi_{\text {taxi }}= & (\tau-d)\left[\theta \int_{0}^{\delta_{T, O}^{A}} \delta f(\delta) d \delta\right. \\
& \left.+(1-\theta)\left(\int_{0}^{\delta_{T, L}^{B}} \delta f(\delta) d \delta+P \int_{\delta_{T, L}^{B}}^{\delta_{L, O}^{B}} \delta f(\delta) d \delta\right)\right]
\end{aligned}
$$

The level of price satisfying the first-order condition ${ }^{6} \partial \Pi_{\text {taxi }} / \partial \tau=0$ is

$$
\tau^{e}=\check{\alpha}+2 d
$$

Condition (4) requires $\check{\triangle}^{e} \geq \kappa^{e}+\frac{1}{2}(\eta+\gamma) x$ and yet $\Delta(\check{\alpha}+d) \geq\{P \eta-(1-\theta) P \beta+[1-\theta(1-P)] \gamma\} x / 2$. It holds according to Assumption 3.

Note that the probability of the bus being on time defined in (7) is continuous.

The core component of the bus fare corresponds to the average taxi trip cost cut by the average schedule and waiting time costs incurred by commuters. The bus company takes account of its service quality to remain attractive regarding the alternative mode. As expected, the punctuality decreases

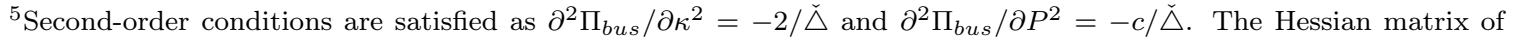
second partial derivatives is also negative definite, and the solution is a global maximum. It satisfies all conditions regarding this maximization problem.

${ }^{6}$ Second-order condition requires that $4 \check{\alpha}-2 \tau^{e}+6 d \geq 0$ or $\tau^{e} \leq 2 \check{\alpha}+3 d$. All conditions are satisfied.
} 
when the punctuality cost $c$ increases. Since the punctuality level decreases with the maximal taxi trip time, $\Delta$, a high scatter of commuters' locations makes the service quality regress (see equation (7)). In addition, the longer is the route where commuters live, the higher is the mark-up for the bus company. The taxi fare is independent of the bus company choices. It only depends on the values of taxi and bus travel time and operating cost. Ceteris paribus when $d$ increases, both bus and taxi fares become higher.

There is a unique simultaneous Nash equilibrium which is given by equations (6), (7) and (8).

Proposition 3. At equilibrium, $P^{e}$, the probability of the bus being on time and $\kappa^{e}$, the bus fare, increase with $\tau$, the taxi fare.

Proof. See AppendixC.

Consider an initial rise in taxi fare, $\tau^{e}$, for example due to an increase in the taxi operating cost or in the gas price if the alternative mode is viewed as the private car. This increase leads to a standard modal shift from taxi service to bus service, other things being equal (see Propositions 1 and 2). Consequently, the cost of the bus punctuality per user decreases. The bus company will therefore have an incentive to increase the punctuality level when $\tau$ rises. By doing so, the bus company attracts additional commuters. In a nutshell, an increase in bus patronage improves the service quality of the bus. This can be viewed as an extension of the Mohring Effect (Mohring, 1972) according to which the service quality measured as the frequency increases when the demand for public transport rises.

The increase in the bus fare is explained by two aspects: on the one hand the service quality has been improved, and on the other hand, the rises in the taxi fare increase the average taxi trip cost and, therefore, the bus fare. This relationship has already been observed, and we refer the reader to some cases where the bus operator has increased the bus fare in response to a rise in gas price (Ya'ar, 2011 and Mohapatra, 2013). There is no strategic complementarity because the taxi company does not take into account the bus fare and service quality.

\section{Welfare analysis}

The social planner maximizes the welfare defined as the sum of the aggregate commuters' and companies' surpluses. Since a cost function is used instead of a surplus function to study the commuter strategies, the social welfare function is defined as the opposite of the social cost function $S C$, which is the difference between aggregate commuter costs and firm profits. From equations of commuter cost (1) and (2), of demand (3a) and (3b), and of companies cost (5a) and (5b), the social cost function can be written as

$$
S C=\frac{\Delta \alpha_{b u s}}{2}+\theta A C C_{A}+(1-\theta) A C C_{B}-\Pi_{b u s}-\Pi_{t a x i},
$$

where $A C C_{i=A, B}$ is the aggregate cost incurred by commuters from Group $i$ such as

$$
\begin{aligned}
A C C_{A}= & (\check{\alpha}+\tau) \int_{0}^{\delta_{T, O}^{A}} \delta f(\delta) d \delta \\
& +\{\kappa+[(1-P)(\eta+\gamma)] x\} \int_{\delta_{T, O}^{A}}^{\Delta} f(\delta) d \delta
\end{aligned}
$$


and

$$
\begin{aligned}
A C C_{B}= & (\check{\alpha}+\tau) \int_{0}^{\delta_{T, L}^{B}} \delta f(\delta) d \delta \\
& +\int_{\delta_{T, L}^{B}}^{\delta_{L, O}^{B}}[(1-P) \kappa+P(\check{\alpha}+\tau) \delta] f(\delta) d \delta \\
& +\{\kappa+[(1-P) \eta+P \beta] x\} \int_{\delta_{L, O}^{B}}^{\Delta} f(\delta) d \delta .
\end{aligned}
$$

The first term in the social cost formula, $\Delta \alpha_{b u s} / 2$, is an unavoidable commuter cost associated to the journey time, recall the normalization we use $\check{\alpha}=\alpha_{\text {taxi }}-\alpha_{\text {bus }}$ leading to Equations (1) and (2).

The social planner chooses the punctuality level $P$, the bus fare $\kappa$ and taxi fare $\tau$ so as to minimize social cost. The first-order conditions for the socially optimal bus and taxi prices are given by

$$
\begin{aligned}
\kappa^{o} & =0 \\
\tau^{o} & =d .
\end{aligned}
$$

As expected, optimal bus and taxi fares equal to the marginal costs incurred by bus and taxi companies. Indeed, as there is no variable cost for the bus, the optimal bus fare is null. The optimal taxi fare is lower than the equilibrium one, meaning that taxi travel should subsidized. This is in line with Arnott (1996) who showed that thanks to economies of density, doubling trips and taxis by means of subsidy reduces the waiting time and increases the social surplus.

The expression of the optimal punctuality level $P^{o}$ is not explicit in the general case because the equation to solve is a cube root i.e it has three solutions with only one real.

$$
P^{o}=\underset{P \in\left[\frac{1}{2} ; 1\right]}{\arg \min } S C .
$$

However, in the extreme case where $\theta=1$, there exists a unique solution ${ }^{7}$ satisfying $\partial S C_{\theta=1} / \partial P=$ 0 . By using (10) and (11), we obtain, for Group $A$

$$
P_{\theta=1}^{o}= \begin{cases}\frac{1}{2} & \text { if } c>c_{2 ; \theta=1}^{o} \\ \frac{\left[\check{\Delta}^{o}-(\eta+\gamma) x\right](\eta+\gamma) x}{c \check{\triangle}^{o}-[(\eta+\gamma) x]^{2}} & \text { if } c \in\left[c_{1 ; \theta=1}^{o} ; c_{2 ; \theta=1}^{o}\right] \\ 1 & \text { if } c<c_{1 ; \theta=1}^{o},\end{cases}
$$

where $\check{\Delta}^{o}=\Delta\left(\check{\alpha}+\tau^{o}\right), c_{1: \theta=1}^{o} \equiv(\eta+\gamma) x$, $c_{2 ; \theta=1}^{o} \equiv\left[2 \check{\triangle}^{o}-(\eta+\gamma) x\right](\eta+\gamma) x / \check{\triangle}^{o}$ and where $c_{1 ; \theta=1}^{o}<c_{2 ; \theta=1}^{o}$. Note that the probability of the bus being on time when $\theta=1$ is continuous.

We generalize the above result to the other extreme case where $\theta=0$ in the following conjecture.

Conjecture 1. For Group B $(\theta=0)$, the punctuality level of the bus $P_{\theta=0}^{o}$ weakly decreases when the

\footnotetext{
${ }^{7}$ Second-order condition is verified as $c \check{\triangle}^{o} \geq[(\eta+\gamma) x]^{2}$.
} 
cost of reliability c increases. There are two critical values of $c, c_{1 ; \theta=0}^{o}$ and $c_{2 ; \theta=0}^{o}$ with $c_{1 ; \theta=0}^{o} \leq c_{2 ; \theta=0}^{o}$ such that:

$$
P_{\theta=0}^{o}= \begin{cases}\frac{1}{2} & \text { if } c>c_{2 ; \theta=0}^{o} \\ 1 & \text { if } c<c_{1 ; \theta=0}^{o}\end{cases}
$$

with $c_{1 ; \theta=0}^{o}<c_{2 ; \theta=0}^{o}$.

Equations (10), (11) and (12) provide the values at optimum in the general case. Equations (14) and (13) point out the optimal punctuality level in extreme cases.

The optimal probability of the bus being on time has the same properties we describe in Section 3: it decreases when the punctuality cost $c$ or the travel time of the commuter living the farthest $\Delta$ increases. The important observation is that the optimal probability of the bus being on time does not necessarily equal to 1 . It may be lower than 1 and even equal to $1 / 2$ under some conditions. Critical values $c_{1 ; \theta=0}^{o}$ and $c_{2 ; \theta=0}^{o}$ are expected because $P_{\theta=0}^{o} \in[1 / 2 ; 1]$. The above conjecture is illustrated in Figure 4 .

From now on, as the expression of $P^{o}$ is not explicit and $P^{o}=\theta P_{\theta=1}^{o}+(1-\theta) P_{\theta=0}^{o}$, properties of the optimal probability of the bus being on time will be addressed separately according to the structure of the population. The two extreme cases $\theta=1$ and $\theta=0$ are highlighted, even if $\theta \geq 1 / 2$ is assumed.

Proposition 4. For Group A $(\theta=1)$, the punctuality level of the bus is higher at optimum than at equilibrium.

Proof. See AppendixE.

Commuters in Group $A$ want to arrive at $T$; therefore, the later bus arrives, the more cost commuters incur. The bus company wishes to maximize the probability of the bus being on time at equilibrium, as the social planner does at optimum, while taking into account the punctuality cost per user incurred by the bus company. The difference between equilibrium and optimum bus punctuality is mainly explained by a price-effect. The gap between the bus fare relative to the taxi fare is much higher at equilibrium than at optimum. Thus, other things being equal, the bus company attracts less customers at equilibrium than at optimum. Consequently, the bus company has to reduce the bus punctuality at equilibrium more than the social planner does at optimum to keep the punctuality cost per user small enough. This result is summarized in Proposition 4.

As there is no explicit expression for $P^{o}$ and $P_{\theta=0}^{o}$, a discussion with a figure is provided in Section 5.

Proposition 5. For Group $A(\theta=1)$, if the taxi operating cost $d$ is higher than $d_{1}^{c}$, the bus patronage is higher at optimum than at equilibrium.

When $d \leq d_{1}^{c}$, the bus patronage is higher at optimum than at equilibrium if and only if the cost of punctuality for the bus company is small enough $\left(c \leq c_{1}^{c}\right){ }^{8}$

\footnotetext{
${ }^{8}$ The critical value of the taxi operating cost $d$ is $d_{1}^{c}=\frac{3(\eta+\gamma) x}{8 \Delta}-\check{\alpha}$. The critical value of the punctuality cost $c_{1}^{c}$ is defined as the unique solution of $D_{\theta=1}^{o}=D_{\theta=1}^{e}$.
} 
Proof. See AppendixF.

As the expression of $P_{\theta=0}^{o}$ is not explicit, the analysis is more difficult for Group B. However, we formulate a proposition as well as a conjecture.

Proposition 6. For Group $B(\theta=0)$, if the taxi operating cost $d$ is lower than $d_{2}^{c}$ (higher than $d_{3}^{c}$, resp.), the bus patronage is lower (resp. higher) at optimum than at equilibrium. ${ }^{9}$

Proof. See AppendixG.

We conjecture the variations in demand for Group $B$ when $d \in\left[d_{2}^{c} ; d_{3}^{c}\right]$.

Conjecture 2. For Group B, when $d \in\left[d_{2}^{c} ; d_{3}^{c}\right]$, the bus patronage is higher at optimum than at equilibrium if the punctuality cost for the bus company is small enough $\left(c>c_{2}^{c}\right){ }^{10}$

This conjecture is discussed in AppendixH. The basic idea in Propositions 5 and 6 and in Conjecture 2 is that when the taxi operating cost is small, the bus company tends to under-price, which consequently attracts too many customers. As the taxi operating cost is high, the bus company overprices. This is due to the fact that the bus fare highly depends on the taxi fare (see equation (6)).

The equilibrium modal split meets the optimal modal split under two conditions. First, the taxi operating cost $d$ has to be included between the two critical values we defined. Then the punctuality cost incurred by the bus company $c$ has to be equal to a critical value. If the taxi operating cost is higher than the interval defined by critical values, the optimal modal split is reached by a partial commuters shift from taking a taxi to taking a bus. This shift can also be in the opposite direction if the taxi operating cost is smaller than the critical interval. This reflects the fact that the bus company under-provides quality relative to the social optimum when $c$ is small.

The taxi operating cost corresponds to the traditional costs as fuel or insurance, but it may also be viewed as an extra tax set by the planner to account for the externalities such as pollution or noise. ${ }^{11}$ In this sense, the operating cost trend should be growing, and in the long run, the bus patronage would increase at the expense of the taxi service.

\section{Numerical application}

We develop an applied case to illustrate previous theoretical findings. Numerical results are obtained with values specified in Table 1 . In the studied case, the bus has a probability $P$ of being on time and a probability $1-P$ of being 10 minutes late at departure. Commuters living the farthest from their trip destination have a taxi trip time equal to 35 minutes. We consider a uniform distribution of the taxi trip time. The operating taxi cost $d$ is constant and equal to $40 \$ /$ hour. Lastly, cost parameters $\alpha_{\text {bus }}, \alpha_{\text {taxi }}, \eta, \beta$ and $\gamma$ are equal to $15,4,20,10$ and $30 \$$ /hour, resp. Each variable is drawn depending on the reliability cost for the bus $c$. Used values are consistent with results from Wardman (2004).

\footnotetext{
${ }^{9}$ The critical values of the taxi operation cost $d$ are $d_{2}^{c}=-\left(\frac{1}{2} \eta-\frac{7}{2} \beta\right) x / 2 \Delta-\alpha_{\text {taxi }}$ and $d_{3}^{c}=(2 \eta+\beta) x / 2 \Delta-\alpha_{\text {taxi }}$, with $d_{2}^{c}<d_{3}^{c}$.

${ }^{10}$ The critical value of reliability $c_{2}^{c}$ is supposed to be the unique solution of $D_{\theta=0}^{o}=D_{\theta=0}^{e}$. We do not prove the
} 


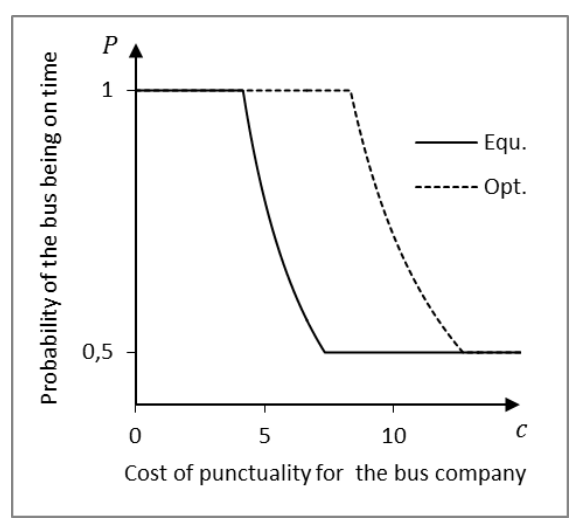

$\operatorname{Group} A(\theta=1)$

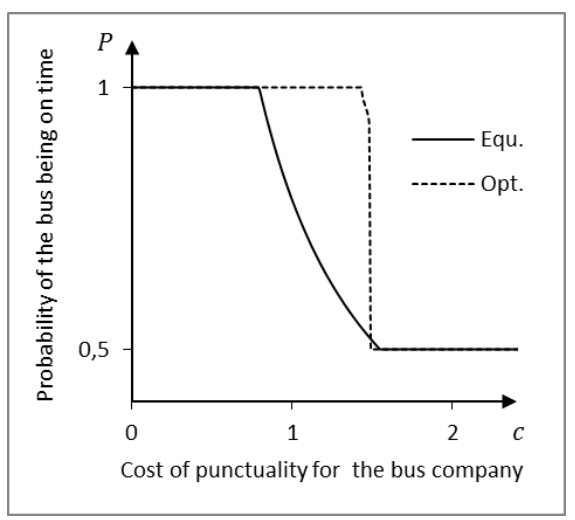

Group $B(\theta=0)$

Figure 4: Probability of the bus being on time as a function of the punctuality $\operatorname{cost} c$

A reminder to the readers, $P^{e}$ and $P^{o}$ are respectively the probability of the bus being on time at equilibrium and at optimum. As expected, the probability of the bus being on time decreases when the reliability cost increases (see Figure 4). The more expensive the punctuality is, the less interesting is the reliability for both the bus company and the social planner.

As indicated in Proposition 4, the probability of the bus being on time when $\theta=1$ is higher at optimum than at equilibrium. The opposite extreme case where $\theta=0$ is more complex as $P^{o}$ is not continuous. It seems that the probability of the bus being on time is higher at optimum than at equilibrium when $c$ is small, and that after a critical value of $c$ this relation is inverted. Probabilities of the bus being on time are higher when $\theta=1$ rather than when $\theta=0$. This is due to the fact that users from Group $A$ are more sensitive to unreliability because when the bus is late they incur a late delay cost which is higher than the waiting time cost incurred by commuters from Group B. Thus when $\theta=1$, the bus company needs to maintain a better level of service than when $\theta=0$ in order to keep their patrons. An important observation is that the optimal punctuality may be very low and even equal to $1 / 2$, which is the worst reliability level. Indeed, since the reliability cost is not too high, the social planner makes the bus company increase the punctuality of the bus to minimize the cost born by users. However, if the punctuality cost for the bus is too high, it is socially better to share cost with users by making or allowing the bus to be late.

Two points are especially interesting in Figure 5. First, the bus patronage is weakly decreasing when the punctuality cost increases. This drop is higher at optimum than at equilibrium. Along with Figure 4 we note that the punctuality has a strong effect on demand. The variations of the bus patronage corresponds to the variations of the bus punctuality. When the bus punctuality is stable, the split between the bus and the taxi is constant. Secondly, note that the demand for the bus is higher at optimum than at equilibrium in both extreme cases. Regarding Proposition 5, this example illustrates the common case where the bus patronage is higher at optimum than at equilibrium.

\footnotetext{
monotonicity of $D_{\theta=0}^{o}-D_{\theta=0}^{e}$, but the study of the boundary cases ( $c$ small and $c$ large) illustrates this conjecture.

${ }^{11}$ We refer the reader to Proost and Van Dender (2001) for an evaluation of alternative fuel efficiency, environmental and transport policies regarding atmospheric pollution.
} 


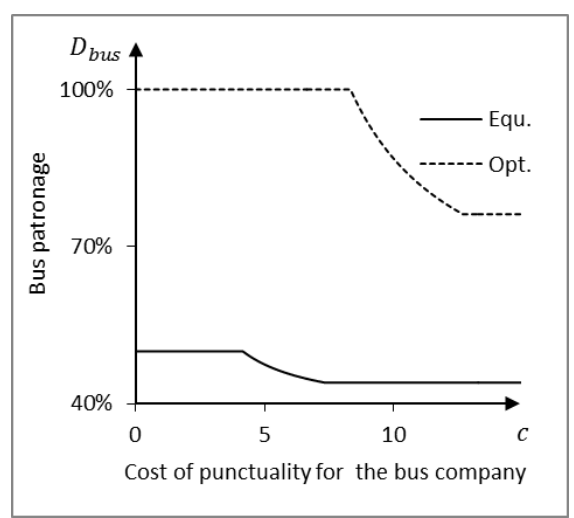

Group $A(\theta=1)$

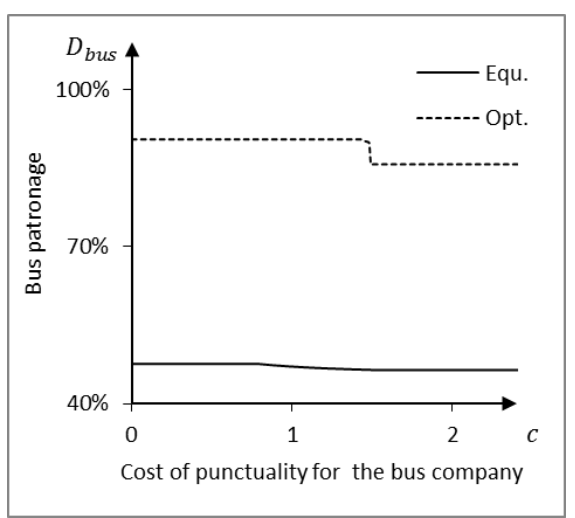

Group $B(\theta=0)$

Figure 5: Bus patronage as a function of the punctuality cost $c$

\begin{tabular}{|c|c|c|c|c|}
\hline Variable & Equilibrium & $\begin{array}{c}\text { First-best } \\
\text { Optimum }\end{array}$ & Decentraliz. & $\begin{array}{c}\text { Second-best } \\
\text { Optimum }\end{array}$ \\
\hline \hline $\begin{array}{c}\text { Probability of the } \\
\text { bus being on time }\end{array}$ & 0.72 & 0.88 & 0.88 & 0.88 \\
\hline Bus fare & $16.4 \$$ & 0 & $16.9 \$$ & 0 \\
\hline Taxi fare & $70 \$ /$ hour & $40 \$ /$ hour & $70 \$ /$ hour & $70 \$ /$ hour \\
\hline Bus patronage & $47 \%$ & $98 \%$ & $50 \%$ & $96 \%$ \\
\hline Social gain & - & $42.1 \%$ & $-13 \%$ & $41.3 \%$ \\
\hline
\end{tabular}

Table 2: Values of main variables when $c=5$ and $\theta=0.75$

The bus patronages is sub-optimal at equilibrium. Too much commuters use the taxi service because catching the bus is too expensive, and the bus is not reliable enough.

Table 2 provides the values of main variables when $c=5$ and $\theta=0.75$ at equilibrium, first-best optimum, decentralized optimum and second-best optimum. The optimum is reached by increasing the reliability and decreasing prices if possible. Consequently, the bus patronage becomes much higher, and the social gain is about $42 \%$.

Usually, the public transport infrastructure has large fixed costs. This leads the social planner to subsidize the operator activity. As the equilibrium probability of the bus being on time is not optimal, one needs an incentive contract between the social planner and the transport operator such as the subsidy depends on the service quality. In particular, from (7) we set

$$
c=\frac{\left[\check{\Delta}^{e}-\left(\eta+\theta \gamma-P^{e} \check{\eta}\right) x\right] \check{\eta} x}{2 \check{\Delta} P^{e}} \text { and } c^{\prime}=\frac{\left[\check{\Delta}^{e}-\left(\eta+\theta \gamma-P^{o} \check{\eta}\right) x\right] \check{\eta} x}{2 \check{\Delta} P^{o}} .
$$

The "optimal" subsidy, $s^{o}$, is such as the punctuality is the same at equilibrium and at optimum. Then,

$$
s^{o} \equiv \frac{\left[\check{\Delta}^{e}-(\eta+\theta \gamma) x\right] \check{\eta} x}{2 \check{\Delta}}\left(\frac{P^{o}-P^{e}}{P^{o} P^{e}}\right) \frac{P^{2}}{2} .
$$

The insufficient provision of reliability due to some monopoly power has been taken into account by the social planner (see column 4 in Table 2). However, there is here a social loss due to the subsidy. 


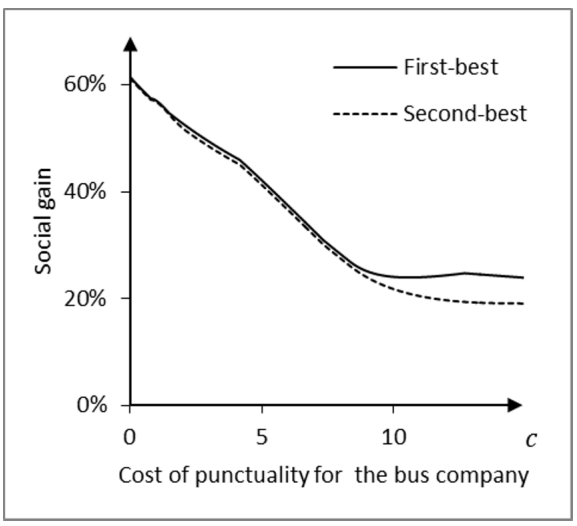

Figure 6: Relative social gains compared to equilibrium as a function of the cost of punctuality $c$ when $\theta=0.75$.

Since subsidy induces the public transport operator to improve the probability of the bus being on time, the bus fare is also raised. There is a transfer of the punctuality cost from the bus company to the social planner and to the users via the subsidy. In this case, the bus company extracts much of the rent and the decentralization is sub-optimal.

The second-best optimum is reached when the taxi service is not optimally priced while the bus company fare and punctuality are. The probability of the bus being on time at equilibrium equals to 0.72 (note that this measure is consistent with observed average lateness in Paris Area (STIF, 2013a)). The bus fare may seem high, but it is not surprising as we do not take into account subsidies which are important in the public transport sector (Ponti, 2011). For example in Paris Area in 2010, monetary public transport revenues equal to $29.7 \%$ of total operating cost (STIF, 2013b). Even if the social planner cannot affect the taxi fare, a second-best optimum may be reached by optimizing both the probability of the bus being on time and the bus fare. The bus patronage is higher than the first-best optimal one because the taxi fare is higher than its optimal value. However, the difference in patronage is small, and the social planner efficiency is not much altered by the competitive taxi fare.

The relative social gains drawn in Figure 6 are computed as the ratio of the absolute gain, due to the transition from equilibrium to optima, to the absolute social cost at equilibrium (see Figure 6). Such curves allow to determine when the gain is high enough to justify public intervention: the lower the punctuality cost is, the more useful is public intervention. When $c$ is high (see equations 7, 13 and 14), punctuality at equilibrium and at optimum is almost the same. The only difference between equilibrium and optima is the modal split, but the gain due to this difference is gradually offset by the growing punctuality cost. Here, once more the difference between first and second-best optima is small but increases with $c$.

The brief application in this section illustrates that the effectiveness of public intervention varies according to the punctuality cost but that the potential gain is still significant. We note that the gap between second-best and first-best optima is very small. It means that the social planner does not need to control the taxi service to be efficient. 


\section{Extension: A second bus is available}

So far it has been assumed that commuters missing the bus leave the bus stop to use the taxi service. However, it seems reasonable to envisage that commuters missing the first bus have the possibility to wait for the next bus. It is now assumed that a second bus arrives "on average" at time $T+h$, with $h>x$. Suppose then that commuters missing the first bus wait for the next one and that by doing so do not use taxis. It means, as before, that commuters have the choice between bus and taxi at the beginning of the journey. However here, a person missing the bus waits for the next bus and does not need, as before, to take a taxi in this case. In this extension, both buses belong to the same bus company which acts as a monopoly. This hypothesis seems realistic as in most urban areas, an unique operator is in charge of the same line. This means that the bus company sets an unique fare for both buses.

\subsection{Demand for buses and taxi}

Three strategies are still available: a commuter may choose the taxi (Strategy $T$ ) or arrive on time at the bus stop (Startegy $O$ ) as before, and he may arrive late at the bus stop and wait for the next bus if he misses the first one (Strategy $L$ ). The generalized costs linked to Strategy $O$ and $T$ remain unchanged from Section 2. Only the cost of choice of Strategy $L$ is changed. The choices of commuters depend on $h$, the time gap between the two buses. If this gap is huge, no-one will take the risk to miss the bus and to wait a very long time for the next one.

Proposition 7. The commuter $\delta$ in Group A selects:

$$
\begin{gathered}
\text { Strategy } O \text { (time } T) \quad \text { if } \quad \delta \geq \delta_{T, O}^{A}, \\
\text { Strategy } T \text { (taxi) } \quad \text { if } \delta<\delta_{T, O}^{A},
\end{gathered}
$$

where $\delta_{T, O}^{A} \equiv[\kappa+(1-P)(\eta+\gamma) x] /(\check{\alpha}+\tau)$.

Proof. See AppendixI.

Proposition 7 is identical to Proposition 1. This is not surprising as users from Group $A$ have no interest to arrive late at the bus stop because this guarantees lateness for them.

Proposition 8. The commuter $\delta$ in Group B selects:

$$
\begin{aligned}
& \text { Strategy } O \text { (time T) if } \quad \delta \geq \delta_{T, O}^{B} \text { and } h>h^{c} \text {, } \\
& \text { Strategy } L \text { (time } T+x) \text { if } \delta \geq \overline{\delta_{T, L}^{B}} \text { and } h \leq h^{c} \text {, } \\
& \text { Strategy } T \text { (taxi) if }\left\{\begin{array}{l}
\delta<\delta_{T, O}^{B} \text { and } h>h^{c}, \\
\delta<\overline{\delta_{T, L}^{B}} \text { and } h \leq h^{c},
\end{array}\right.
\end{aligned}
$$

where $\delta_{T, O}^{B} \equiv[\kappa+(1-P) \eta x+P \beta x] /(\check{\alpha}+\tau)$,

$\overline{\delta_{T, L}^{B}} \equiv[\kappa+P(\gamma+\eta)(h-x)] /(\check{\alpha}+\tau)$ and

$h^{c} \equiv[1+((1-P) \eta / P+\beta) /(\eta+\gamma)] x$.

Proof. See AppendixJ. 


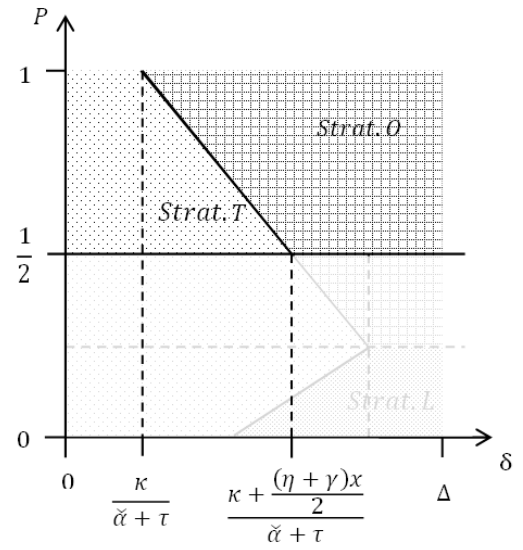

Group A

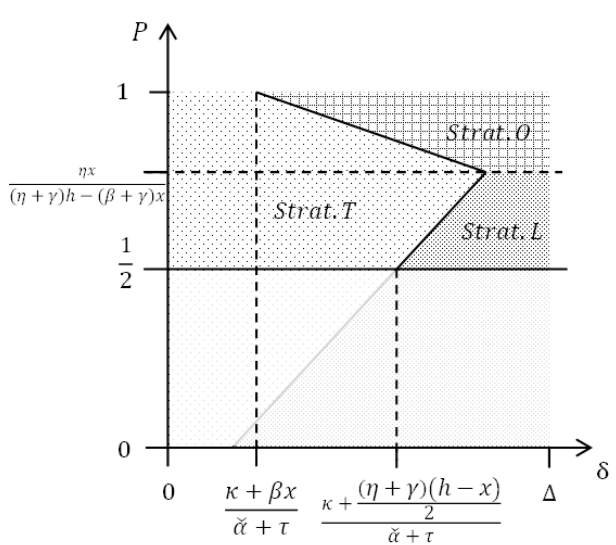

Group B

Figure 7: Strategies spaces as a function of $P$, the probability of the bus being on time.

Among Group B, some commuters choose to arrive late at the bus stop, provided that the next bus does not arrive too late. These commuters are living close to the city center.

In this extension, the share of commuters choosing Strategy $T$ is the taxi patronage and complementarity commuters choosing Strategy $O$ or Strategy $L$ are bus users. Figure 7 illustrates both strategy and patronage shares in Groups $A$ and $B$, depending on the probability of the bus being on time. There is no surprise for Group $A$ : the bus patronage increases with the reliability and reaches its highest level when $P=1^{12}$. The analysis for Group $B$ is quite similar. The bus patronage first decreases linearly with the punctuality, and when the punctuality comes above a threshold value, the bus patronage increases. This threshold embodies the level of punctuality which is the most costly for users. Below this threshold, commuters arrive late at the bus station, and above, they arrive on time.

The bus company still chooses the probability of the bus being on time, $P$, and the price of the ticket, $\kappa . h$ is assumed exogenous. By setting the punctuality level relatively to $h$, the bus company decides if users from Group B arrive on time or late at the bus station. Figure 8 shows which values of $P$ allow the bus company to induce users to arrive on time (or late) at the bus stop. The bus company is not able to make all users arriving at the bus station at time $T+x$ because $h$ has to be higher than $x$ and $P$ higher than $1 / 2$. There are three different situations depending on $h$. When $h \geq[1+(\eta+\beta) /(\eta+\gamma)] x$, the second bus arrives too late to be a credible alternative, so all users arrive on time at the bus station to be sure of not missing the first bus, regardless of the punctuality. In the same vein, if $h \leq[1+\beta /(\eta+\gamma)] x$, then users from Group B arrive late at the bus stop. Indeed, by doing so, they incur no schedule or waiting cost if they manage to get the first bus, and if they miss it, the next one arrives so early that the incurred costs are negligible. When $h$ is between this two extreme cases, the bus company chooses to induce users from Group B to arrive on time or late by setting the level of the probability of the bus being on time. This strategy maximizes its profit. The strategies defined above give the demand functions.

\footnotetext{
${ }^{12}$ If a probability lower than one half had been considered, the analysis would have been different. When the punctuality is getting close to its lowest level $(P=0)$, the bus patronage increases again. This reflects the fact that when the bus is almost always late, it is in fact more regular to the commuters.
} 


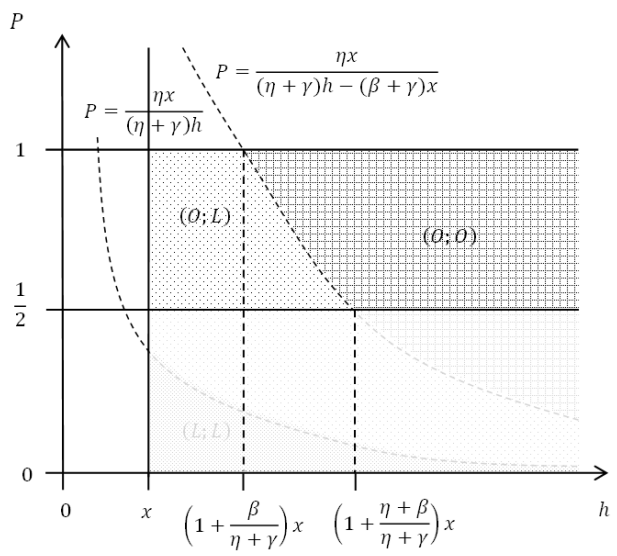

Figure 8: Map of commuter's strategies depending on the probability of the bus being on time, $P$, and the arrival time of the next bus, $h$, where (I,J) is strategy of Group $A(\mathrm{I})$ and strategy of Group B (J).

The expression of the demands for buses and for taxi depend on the value of $h$ :

$$
\begin{aligned}
& D_{\text {buses }}= \begin{cases}1-\frac{\theta \delta_{T, O}^{A}+(1-\theta) \delta_{T, O}^{B}}{\Delta} & \text { if } h>h^{c} \\
1-\frac{\theta \delta_{T, O}^{A}+(1-\theta) \overline{\delta_{T, L}^{B}}}{\Delta} & \text { if } h \leq h^{c}\end{cases} \\
& D_{\text {taxi }}= \begin{cases}\frac{\theta \delta_{T, O}^{A}+(1-\theta) \delta_{T, O}^{B}}{\Delta} & \text { if } h>h^{c} \\
\frac{\theta \delta_{T, O}^{A}+(1-\theta) \overline{\delta_{T, L}^{B}}}{\Delta} & \text { if } h \leq h^{c}\end{cases}
\end{aligned}
$$

Note that the demand for buses is linear with the bus fare whereas the taxi patronage is convex depending on the taxi fare.

\subsection{Competition}

The cost production functions are still defined by Equations (5a) and (5b). The expressions of profit are different in this extension from those of the main model, because commuters missing the first bus take the second bus instead of using taxi. From Equations (15a) and (15b), taxi and bus company profit function are given by

$$
\begin{gathered}
\frac{\Pi_{\text {taxi }}}{\tau-d}=\left\{\begin{array}{cc}
\theta \int_{0}^{\delta_{T, O}^{A}} \delta f(\delta) d \delta+(1-\theta) \int_{0}^{\delta_{T, O}^{B}} \delta f(\delta) d \delta & \text { if } h>h^{c}, \\
\theta \int_{0}^{\delta_{T, O}^{A}} \delta f(\delta) d \delta+(1-\theta) \int_{0}^{\overline{\delta_{T, L}^{B}}} \delta f(\delta) d \delta & \text { if } h \leq h^{c}
\end{array}\right. \\
\Pi_{\text {bus }}=\kappa D_{\text {bus }}-\frac{c}{2} P^{2},
\end{gathered}
$$

where $\delta_{T, O}^{A}, \delta_{T, O}^{B}, \overline{\delta_{T, L}^{B}}$ and $h^{c}$ are defined in Propositions 7 and 8.

The taxi company decides about the optimal fare so as to maximize its profit. The first-order 
condition gives ${ }^{13}$

$$
\tau^{e}=\check{\alpha}+2 d .
$$

The optimization of the bus company profit needs to be treated in two distinct cases according to the position of $h$ with respect to $h^{c}$. We present the detailed results in AppendixK. The equilibrium bus fare has the same structure than the one described in Equation (6). It corresponds to the average taxi trip cost cut by the average schedule and waiting time costs incurred by commuters. The probability of the bus being on time equals to 1 when $c$ is smaller than a low threshold, $1 / 2$ when $c$ is above an upper threshold. Between these two thresholds, the probability is continuous and decreasing with respect to $c$.

When $h^{c} \leq h$, the results and properties of the equilibrium are the same than the ones displayed when only one bus was available (see Section 3). When $h$ is small, the reliability is a decreasing function of $h$. Until a critical arrival time of the next bus (beyond which all users are punctual), when $h$ increases, users become more captive to the first bus, and then, the reliability of this bus may deteriorate.

Proposition 9. At equilibrium, $P^{e}$, the probability of the bus being on time and $\kappa^{e}$, the bus fare, increase with $\tau$, the taxi fare.

Proof. See AppendixM.

When $h \in[x ;[1+\beta /(\eta+\gamma)] x] \cup[[1+(\eta+\beta) /(\eta+\gamma)] x ; \infty]$, the bus company has no influence in the type of strategy chosen by bus users. However, when $h \in] x ;[1+\beta /(\eta+\gamma)] x[$, it may set the bus fare and punctuality such as users in Group B choose Strategy $O$ or Srategy L. This decision maximizes the bus company's profit. ${ }^{14}$.

\subsection{Welfare analysis}

The optimal situation is obtained by minimizing the social cost function described in equation (9). The first-order condition for this problem is

$$
\begin{aligned}
& \kappa^{o}=0 \\
& \tau^{o}=d .
\end{aligned}
$$

Equations (19) and (20) are standard results stating that optimal prices must equal marginal cost of production. The optimization of the reliability level must be treated in two separate cases depending on the value of $h$ relative to the critical value $h^{c}$. We display the detailed results in AppendixL. Once $h$ is higher than $h^{c}$, the service quality does not depend on $h$ anymore, because all users are captive to the first bus. In both cases punctuality decreases with the punctuality cost, $c$, and the travel time of the commuter living farthest, $\Delta$.

\footnotetext{
${ }^{13}$ Second-order condition is verified for any value of $h$.

${ }^{14}$ We do not focus on this issue because on the one hand, the interval is very small (equal to $\eta /(\eta+\gamma)$ ), and, on the other hand, it has no effect on the main results.
} 


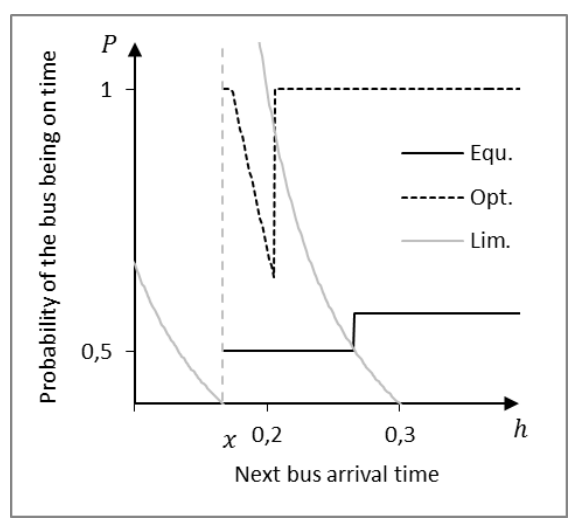

$c=4$ and $\theta=0.5$

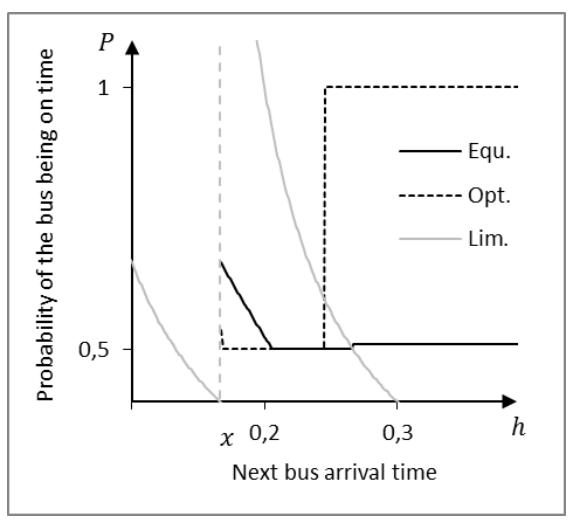

$c=3$ and $\theta=0.25$

Figure 9: Probability of the bus being on time as a function of the next bus arrival time $h$.

Proposition 10. When $h \in[x ;[1+\beta /(\eta+\gamma)] x] \cup[[1+(\eta+\beta) /(\eta+\gamma)] x ; \infty]$, the punctuality level of the bus is higher (lower, resp.) at optimum than at equilibrium when $c>c^{c}\left(c \leq c^{c} \text {, resp. }\right)^{15}$.

Proof. See AppendixN.

The intuition behind this proposition is that sometimes, when the punctuality cost is small, then the bus company sets a higher level of punctuality than at optimum to raise the patronage for buses. The increase in cost is more than offset by the increase in revenue due to the higher patronage. However in most cases, the punctuality is too low at equilibrium because as users living far from the city center are somehow captive of bus, the level of punctuality may be deteriorated. The increase of patronage due to a service quality improvement does not offset the increase in cost.

We use the values of parameters defined in Table 1 to draw Figure 9. Both graphs show variations of the punctuality depending on the time gap between buses. In the first situation, when $h$ is small, users from Group $B$ arrive late at the bus station. The bus company gives priority to them by setting a very low level of punctuality. At the time $h$ goes over $h^{c}$, the punctuality increases because all users arrive on time at the bus station. This graph is a typical case where the optimal service quality is still higher than the equilibrium one. The second graph points out a more paradoxical case where the two curves cross. Commuters from Group B are three times more than from Group A. This explains why both curves are drown to the bottom when users from Group $B$ arrive late at the station. When $h$ is higher, the optimal punctuality equals to 1 whereas the equilibrium service quality remains low. A degraded service is not so penalizing for users from Group $B$ who are the majority. When $h$ stands below the threshold, the punctuality is higher at equilibrium than at optimum.

To summarize, this extension strengthens our main findings, in particular the Mohring Effect which arises once again. Moreover, we show that the frequency matters as it makes users punctual or not at the bus station.

\footnotetext{
${ }^{15}$ The critical value of the bus punctuality $\operatorname{cost} c^{c}$ is defined in AppendixN.
} 


\section{Conclusion}

The modeling of the bus punctuality reported here has provided an improved understanding of the two-way implication between punctuality level of public transport and customer public transport use. Commuters develop adaptive strategies to fit the transport system. Thus, a rise in the fare of one mode decreases the patronage for this mode. In particular, an increase in the taxi fare rises the share of commuters arriving on time at the bus stop because they wish to minimize the probability of missing the bus. Moreover, when the bus company becomes less strict as regards punctuality, more bus users will prefer to arrive late at the bus stop. Then, the bus company is not incited to maintain a high level of reliability. This can generate a vicious circle. We also appreciate the efficiency of the punctuality when it is viewed as an instrument of service quality that can be adapted to fit and regulate the public transport patronage. We propose an extension to overcome a restrictive hypothesis. This allows us to apprehend the importance of service frequency in the commuters strategies. A new market share of commuters is assailable with a reasonable effort in terms of service quality. Compared with the optimum, buses are very often too late at equilibrium. Commuters bear the cost of this extra-lateness, because they have to wait too much for the bus or take the taxi which is expensive. However, it does not mean that the bus should not be late. Indeed if the cost of the punctuality is too high relative to the cost of the alternative mode, a late bus is socially preferable. Finally, we find that the sign and the amplitude of the gap between the equilibrium and the optimal modal split first depends on the cost of the alternative mode and secondly on the punctuality cost incurred by the bus company. Nevertheless, in the more general and realistic case the bus patronage seems under-optimal.

Several elements remain to be addressed. Risk averse users would change users' strategies and affect the punctuality. It should be interesting to include congestion on road networks and in the bus. Congestion on the road would make the taxi journey longer and unpredictable, whereas congestion in the bus (understood as crowding) would accentuate the cost incurred by users. Finally, introducing the bus punctuality in a bus transit line with several stops and several buses (de Palma and Lindsey, 2001) will improve the modeling by introducing a snowball effect: if a bus is late, its lateness increases along its journey.

\section{Acknowledgments}

We are grateful to Simon P. Anderson, Robin C. Lindsey, Stef Proost, Nathalie Picard and two anonymous reviewers for useful comments and to the participants of the WEPSeminar at the ENS Cachan. We thank the Co-Editor Gilles Duranton for insightful advices and suggestions.

\section{References}

Arnott, R., 1996. Taxi travel should be subsidized. Journal of Urban Economics 40 (3), 316-333.

Arnott, R., de Palma, A., Lindsey, R., 1990. Economics of a bottleneck. Journal of Urban Economics $27(1), 111-130$.

Balcombe, R., Mackett, R., Paulley, N., Preston, J., Shires, J., Titheridge, H., Wardman, M., White, P., 2004. The demand for public transport: a practical guide. 
Bates, J., Polak, J., Jones, P., Cook, A., 2001. The valuation of reliability for personal travel. Transportation Research Part E: Logistics and Transportation Review 37, $191-229$.

Beirao, G., Cabral, J. S., 2007. Understanding attitudes towards public transport and private car: A qualitative study. Transport Policy 14 (6), $478-489$.

Coulombel, N., de Palma, A., 2014. The variability of travel time, congestion, and the cost of travel. Mathematical Population Studies, in press.

de Palma, A., Kilani, M., Proost, S., 2013. Discomfort in mass transit and its implication for scheduling and pricing, working paper, ENS Cachan.

de Palma, A., Lindsey, R., 2001. Optimal timetables for public transportation. Transportation Research Part B: Methodological 35 (8), $789-813$.

Fosgerau, M., Engelson, L., Franklin, J. P., 2014. Commuting for meetings, unpublished manuscript.

Fosgerau, M., Karlström, A., 2010. The value of reliability. Transportation Research Part B: Methodological 44 (1), $38-49$.

Hensher, D. A., Stopher, P., Bullock, P., 2003. Service quality - developing a service quality index in the provision of commercial bus contracts. Transportation Research Part A: Policy and Practice 37 (6), $499-517$.

Jensen, M., 1999. Passion and heart in transport - a sociological analysis on transport behaviour. Transport Policy 6 (1), 19 - 33.

Mohapatra, D., 2013. Fuel price rise: City bus fares hiked. The Times of India, 24 September. URL http://articles.timesofindia.indiatimes.com/2013-09-24/bhubaneswar/42359288_1_ fuel-price-rise-city-bus-fares-rs-33

Mohring, H., 1972. Optimization and scale economies in urban bus transportation. The American Economic Review 62 (4), $591-604$.

Paulley, N., Balcombe, R., Mackett, R., Titheridge, H., Preston, J., Wardman, M., Shires, J., White, P., 2006. The demand for public transport: The effects of fares, quality of service, income and car ownership. Transport Policy 13 (4), 295 - 306.

Ponti, M., 2011. A Handbook of Transport Economics. Edward Elgar, Ch. 28 Competition, regulation and public service obligations, pp. 661-683.

Proost, S., Van Dender, K., 2001. The welfare impacts of alternative policies to address atmospheric pollution in urban road transport. Regional Science and Urban Economics 31 (4), 383 - 411.

Redman, L., Friman, M., Gärling, T., Hartig, T., 2013. Quality attributes of public transport that attract car users: A research review. Transport Policy 25 (0), 119 - 127. 
Steinmann, L., 2013. Saturation du trafic sncf : les usagers favorables aux horaires de travail décalés. Les Echos, 2 April.

URL http://www.lesechos.fr/entreprises-secteurs/auto-transport/actu/ 0202676345277-horaires-de-travail-decales-1-idee-de-la-sncf-approuvee/

-par-les-usagers-554261.php

STIF, 2013a. La qualité de service en chiffres - bulletin d'information trimestriel sur la qualité de service des transports en ile-de-france 10.

STIF, 2013b. Le financement du fonctionnement des transports publics.

URL http://www.stif.org/organisation-missions/volet-economique/ financement-transports-publics/financement-transports-franciliens-442.html

Sutton, J., 1991. Sunk costs and market structure: Price competition, advertising, and the evolution of concentration. The MIT press.

Vickrey, W. S., 1969. Congestion theory and transport investment. The American Economic Review $59(2), 251-260$.

Wardman, M., 2004. Public transport values of time. Transport Policy 11 (4), 363 - 377.

Ya'ar, C., 2011. Leap in gasoline prices hits commuters where it hurts. Israel National News.

URL http://www.israelnationalnews. com/News/News.aspx/141479\#.UpXmisTuJqU 


\section{AppendixA. Proof of Proposition 1}

We wish to compare expected costs of Strategys $A, B$ and $O$, denoted $E C(O), E C(L)$ and $E C(T)$ respectively, to define a choice rule for a commuter in Group A. From equations (1) and (2), we can write:

$$
\begin{aligned}
& E C(O)=\kappa+(1-P)(\eta+\gamma) x, \\
& E C(L)=P \delta(\check{\alpha}+\tau)+(1-P)(\kappa+\gamma x), \\
& E C(T)=\delta(\check{\alpha}+\tau)
\end{aligned}
$$

Therefore we have

$$
\begin{gathered}
E C(O) \leq E C(L) \text { iff } \delta \geq \frac{\kappa+\left(\frac{1-P}{P}\right) \eta x}{\check{\alpha}+\tau} \equiv \delta_{L, O}^{A}, \\
E C(T)<E C(L) \text { iff } \delta<\frac{\kappa+\gamma x}{\check{\alpha}+\tau} \equiv \delta_{T, L}^{A}, \\
E C(T)<E C(O) \text { iff } \delta<\frac{\kappa+(1-P)(\eta+\gamma) x}{(\check{\alpha}+\tau)} \equiv \delta_{T, O}^{A} .
\end{gathered}
$$

We use Assumption.1 and Assumption.2 to rank $\delta_{L, O}^{A}, \delta_{T, L}^{A}$ and $\delta_{T, L}^{A}$ :

(i) $\delta_{T, L}^{A} \geq \delta_{L, O}^{A} \Longleftrightarrow \gamma \geq\left(\frac{1-P}{P}\right) \eta \Longleftrightarrow \frac{\gamma}{\eta} \geq \frac{1-P}{P}$ which is true since $\frac{\gamma}{\eta}>1 \geq \frac{1-P}{P}$ by Assumption.1 and Assumption.2.

(ii) $\delta_{T, L}^{A} \geq \delta_{T, O}^{A} \Longleftrightarrow \gamma \geq(1-P)(\eta+\gamma) \Longleftrightarrow P \geq 1-\frac{\gamma}{(\eta+\gamma)}$ which is true since $\frac{\gamma}{(\eta+\gamma)}>\frac{1}{2}$ by Assumption.2.

(iii) $\delta_{T, O}^{A} \geq \delta_{L, O}^{A} \Longleftrightarrow \eta+\gamma \geq \frac{1}{P} \eta \Longleftrightarrow P \geq \frac{\eta}{(\eta+\gamma)}$ which is true since $\frac{\eta}{(\eta+\gamma)}<\frac{1}{2}$ by Assumption.2.

Therefore $\delta_{L, O}^{A} \leq \delta_{T, O}^{A} \leq \delta_{T, L}^{A}$.

Strategys $B$ is chosen if and only if $\delta<\delta_{L, O}^{A}$ and $\delta \geq \delta_{T, L}^{A}$. As $\delta_{L, O}^{A} \leq \delta_{T, L}^{A}$, Strategys $B$ is dominated and never chosen by commuter in Group A. Figure A.10 summarizes results of the proof.

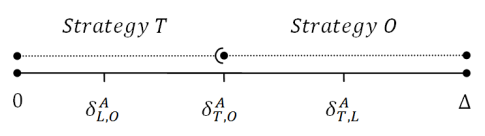

Figure A.10: Strategy choice of a commuter in Group $A$ depending on the taxi trip time $\delta$.

\section{AppendixB. Proof of Proposition 2}

We wish to compare expected costs of Strategys $A, B$ and $O$, denoted $E C(O), E C(L)$ and $E C(T)$ respectively, to define a choice rule for a commuter in Group B. From equations (1) and (2), we can write:

$$
\begin{aligned}
& E C(O)=\kappa+[(1-P) \eta+P \beta] x, \\
& E C(L)=P \delta(\check{\alpha}+\tau)+(1-P) \kappa,
\end{aligned}
$$


$E C(T)=\delta(\check{\alpha}+\tau)$.

Therefore we have

$$
\begin{gathered}
E C(O) \leq E C(L) \text { iff } \delta \geq \frac{\kappa+\left(\frac{1-P}{P} \eta+\beta\right) x}{\check{\alpha}+\tau} \equiv \delta_{L, O}^{B}, \\
E C(T)<E C(L) \text { iff } \delta<\frac{\kappa}{\check{\alpha}+\tau} \equiv \delta_{T, L}^{B}, \\
E C(T)<E C(O) \text { iff } \delta<\frac{\kappa+[(1-P) \eta+P \beta] x}{\check{\alpha}+\tau} \equiv \delta_{T, O}^{B} .
\end{gathered}
$$

We use Assumption.1 and Assumption.2 to $\operatorname{rank} \delta_{L, O}^{B}, \delta_{T, L}^{B}$ and $\delta_{T, L}^{B}$ :

(i) $\delta_{T, L}^{B} \leq \delta_{L, O}^{B} \Longleftrightarrow 0 \leq\left(\frac{1-P}{P} \eta+\beta\right)$ which is true.

(ii) $\delta_{T, L}^{B} \leq \delta_{T, O}^{B} \Longleftrightarrow 0 \leq(1-P) \eta+P \beta$ which is true.

(iii) $\delta_{T, O}^{B} \leq \delta_{L, O}^{B} \Longleftrightarrow(1-P) \eta+P \beta \leq \frac{1-P}{P} \eta+\beta \Longleftrightarrow 1 \geq \frac{1}{P}$ which is true since $\frac{1}{P} \geq 1$ by Assumption.1.

Therefore $\delta_{T, L}^{B} \leq \delta_{T, O}^{B} \leq \delta_{L, O}^{B}$. Figure B.11 summarizes results of the proof.

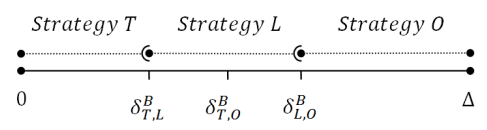

Figure B.11: Strategy choice of a commuter in Group B depending on the taxi trip time $\delta$.

\section{AppendixC. Proof of Proposition 3}

We wish to show that $P^{e}$, the probability of the bus being on time at equilibrium, and $\kappa^{e}$, the bus fare, increase with $\tau$ the taxi fare. We first show that $\partial P^{e} / \partial \tau \geq 0$ (i) and that $\partial \kappa^{e} / \partial \tau \geq 0$ (ii). Then we check that boundaries of interval, $c_{1}^{e}$ (iii) and $c_{2}^{e}$ (iv), increase with $\tau$. Let us recall expressions of equilibrium variables (see equations (6) and (7)):

$$
\begin{aligned}
& \kappa^{e}= \frac{1}{2}\left\{\check{\triangle}^{e}-\left[\left(1-P^{e}\right) \eta+(1-\theta) P^{e} \beta+\theta\left(1-P^{e}\right) \gamma\right] x\right\} \\
& P^{e}= \begin{cases}\frac{1}{2} & \text { if } c>c_{2}^{e}, \\
\frac{\kappa^{e} \check{\eta} x}{c \triangle^{e}} & \text { if } c \in\left[c_{1}^{e} ; c_{2}^{e}\right], \\
1 & \text { if } c<c_{1}^{e},\end{cases}
\end{aligned}
$$


where $\check{\triangle}^{e}=\Delta\left(\check{\alpha}+\tau^{e}\right), \check{\eta}=\eta-(1-\theta) \beta+\theta \gamma, c_{1}^{e} \equiv \kappa^{e} \check{\eta} x / \check{\triangle}^{e}$ and where $c_{2}^{e} \equiv 2 c_{1}^{e}$. By substituting $\kappa^{e}$ in $P^{e}$, we obtain

$$
P^{e}= \begin{cases}\frac{1}{2} & \text { if } c>c_{2}^{e}, \\ \frac{\left[\check{\Delta}^{e}-(\eta+\theta \gamma) x\right] \breve{\eta} x}{2 c \check{\triangle}^{e}-(\check{\eta} x)^{2}} & \text { if } c \in\left[c_{1}^{e} ; c_{2}^{e}\right] \\ 1 & \text { if } c<c_{1}^{e},\end{cases}
$$

where $c_{1}^{e}=\left[\check{\triangle}^{e}-(1-\theta) \beta x\right] \check{\eta} x / 2 \check{\triangle}^{e}$ and where $c_{2}^{e}=\left(\check{\triangle}^{e}-\check{\eta} x\right) \check{\eta} x / 2 \check{\triangle}^{e}$. We now derive $P^{e}, \kappa^{e}, c_{1}^{e}$ and $c_{2}^{e}$ on $\tau^{e}$.

(i) $\frac{\partial P^{e}}{\partial \tau^{e}}=\Delta \check{\eta} x \frac{2 c(\eta+\theta \gamma) x-(\check{\eta} x)^{2}}{\left[2 c \check{\triangle}^{e}-(\check{\eta} x)^{2}\right]^{2}}$ so $\frac{\partial P^{e}}{\partial \tau} \geq 0$ if $c \geq \frac{(\check{\eta} x)^{2}}{2(\eta+\theta \gamma) x}$. Let us substitute $c$ by $c_{1}^{e}$ the minimal value of the interval $\left[c_{1}^{e} ; c_{2}^{e}\right]$. Thus

$$
c_{1}^{e}-\frac{(\check{\eta} x)^{2}}{2(\eta+\theta \gamma) x}=\frac{\check{\eta} x\left[\check{\triangle}^{e}-(\eta+\theta \gamma) x\right](1-\theta) \beta x}{2 \check{\triangle}^{e}(\eta+\theta \gamma) x} .
$$

Yet $\frac{\check{\eta} x(1-\theta) \beta x}{2 \check{\triangle}^{e}(\eta+\theta \gamma) x} \geq 0$ and $\check{\triangle}^{e}-(\eta+\theta \gamma) x \geq 0$ by Assumption.3. We therefore have $\frac{\partial P^{e}}{\partial \tau} \geq 0$;

(ii) $\frac{\partial \kappa^{e}}{\partial \tau^{e}}=\frac{\Delta}{2}+\frac{\partial P^{e}}{\partial \tau^{e}} \frac{\check{\eta} x}{2} \geq 0$ by Assumption.2,

(iii) $\frac{\partial c_{1}^{e}}{\partial \tau^{e}}=\frac{(1-\theta) \beta x \check{\eta} x}{2 \Delta\left(\alpha_{t a x i}+\tau^{e}\right)^{2}} \geq 0$,

(iv) $\frac{\partial c_{2}^{e}}{\partial \tau^{e}}=\frac{(\check{\eta} x)^{2}}{2 \Delta\left(\alpha_{\text {taxi }}+\tau\right)^{2}} \geq 0$.

$P^{e}$, the probability of the bus being on time at equilibrium, and $\kappa^{e}$, the bus fare, increase well with $\tau^{e}$ the taxi fare.

\section{AppendixD. Optimal bus and taxi fare}

The social planner chooses the punctuality level $P$, the bus fare $\kappa$ and taxi fare $\tau$ so as to minimize social cost. The first-order conditions for the socially optimal bus and taxi prices are given by

$$
\begin{aligned}
\frac{\partial S C}{\partial \kappa} & =\frac{\kappa(\check{\alpha}+d)-(\tau-d) \Gamma x}{\Delta(\check{\alpha}+\tau)^{2}}=0, \\
\frac{\partial S C}{\partial \tau} & =\frac{(\tau-d) A-\kappa(\check{\alpha}+d)(\kappa+\Gamma x)}{\Delta(\check{\alpha}+\tau)^{3}}=0,
\end{aligned}
$$

where $\Gamma=(1-P) \eta+(1-\theta) P \beta+\theta(1-P) \gamma, A=\kappa \Gamma x+\chi$ and $\chi=\theta[(1-P)(\eta+\gamma) x]^{2}+(1-\theta) P\left[\left(\frac{1-P}{P} \eta+\beta\right) x\right]^{2}$. Therefore from (D.1a) and (D.1b)

$$
\begin{aligned}
\kappa^{o} & =\frac{\left(\tau^{o}-d\right) \Gamma x}{(\check{\alpha}+d)} \\
\tau^{o} & =\frac{\kappa^{o}(\check{\alpha}+d)\left(\kappa^{o}+\Gamma x\right)}{A \Delta\left(\check{\alpha}+\tau^{o}\right)^{3}}-d .
\end{aligned}
$$


By substituting (D.2a) into (D.2b), the first-best optimal bus and taxi prices can be written as ${ }^{16}$

$$
\begin{aligned}
\kappa^{o} & =0 \\
\tau^{o} & =d .
\end{aligned}
$$

\section{AppendixE. Proof of Proposition 4}

We wish to show that the probability of the bus being on time is higher in the optimal situation than in equilibrium when $\theta=1$. For that, we need to show that the result of $P_{\theta=1}^{o}-P_{\theta=1}^{e}$ is positive (i) and that the limits of the variation intervals are well sorted i.e. $c_{1 ; \theta=1}^{o} \geq c_{1 ; \theta=1}^{e}$ (ii) and $c_{2 ; \theta=1}^{o} \geq c_{2 ; \theta=1}^{e}$ (iii):

(i) $P_{\theta=1}^{o}-P_{\theta=1}^{e}=\frac{c \check{\Delta}(\eta+\gamma) x[\check{\Delta}-(\eta+\gamma) x]}{\left\{2 c \check{\Delta}-[(\eta+\gamma) x]^{2}\right\}\left\{c \check{\Delta}-[(\eta+\gamma) x]^{2}\right\}} \geq 0$ by Assumption.3;

(ii) $c_{1 ; \theta=1}^{o}-c_{1 ; \theta=1}^{e}=\frac{\check{\Delta}(\eta+\gamma) x}{2 \check{\triangle}} \geq 0$ by Assumption.3;

(iii) $c_{2 ; \theta=1}^{o}-c_{2 ; \theta=1}^{e}=\frac{[2 \check{\Delta}-(\eta+\gamma) x](\eta+\gamma) x}{2 \check{\triangle}} \geq 0$ by Assumption.3.

The probability of the bus being on time well and truly is higher in the optimal situation than in equilibrium.

\section{AppendixF. Proof of Proposition 5}

The idea of the proof is that the difference between optimal demand for the bus and equilibrium demand for the bus is a function of $c$, the bus punctuality cost and $d$ the taxi operating cost. Throughout this proof we consider the extreme case where $\theta=1$. Let us recall the expression of demand for the bus function:

$$
D_{b u s}=\int_{\delta_{T, O}^{A}}^{\Delta} f(\delta) d \delta
$$

where $\delta_{T, O}^{A}=[\kappa+(1-P)(\eta+\gamma) x] /(\check{\alpha}+\tau)$. We can define

$$
\begin{aligned}
\bar{D} \equiv D_{\text {bus }}^{o}-D_{\text {bus }}^{e}= & 1-\frac{\kappa^{o}+\left(1-P^{o}\right)(\eta+\gamma) x}{\Delta\left(\check{\alpha}+\tau^{o}\right)} \\
& -\left(1-\frac{\kappa^{e}+\left(1-P^{e}\right)(\eta+\gamma) x}{\Delta\left(\check{\alpha}+\tau^{e}\right)}\right),
\end{aligned}
$$

where $\kappa^{o}=0, \tau^{o}=d, \kappa^{e}=\frac{1}{2}\left[\Delta\left(\check{\alpha}+\tau^{e}\right)-\left(1-P^{e}\right)(\eta+\gamma) x\right]$ and $\tau^{e}=\check{\alpha}+2 d$. We therefore have

$$
\bar{D}=\frac{2 \Delta(\check{\alpha}+d)-\left(3+P^{e}-4 P^{o}\right)(\eta+\gamma) x}{4 \Delta(\check{\alpha}+d)} .
$$

\footnotetext{
${ }^{16}$ Second-order conditions are satisfied as they require $\left(\alpha_{\text {taxi }}+d\right) \geq 0$ and $A \geq 0$.
} 
Since $P^{e}$ and $P^{o}$ are functions of $c$ (equations (7) and (13)), we derive $\bar{D}$ on $c$. For that, we need to know the order of $c_{1}^{e}, c_{2}^{e}, c_{1}^{o}$ and $c_{2}^{o}$. We know that $c_{1}^{e} \leq c_{2}^{e}$ and $c_{1}^{o} \leq c_{2}^{o}$.

$$
\begin{aligned}
c_{1}^{o}-c_{2}^{e} & =(\eta+\gamma) x-\frac{2 \kappa^{e} \check{\eta} x}{\check{\triangle}^{e}} \\
\Longleftrightarrow \quad c_{1}^{o}-c_{2}^{e} & =(\eta+\gamma) x\left[1-\frac{(1-P) 2 \kappa^{e}}{\check{\triangle}^{e}}\right], \\
\Longleftrightarrow \quad c_{1}^{o}-c_{2}^{e} & =(\eta+\gamma) x\left[1-(1-P)\left(1-\frac{\Gamma^{e} x}{\check{\triangle}^{e}}\right)\right] \geq 0 .
\end{aligned}
$$

We therefore have $c_{1}^{e} \leq c_{2}^{e} \leq c_{1}^{o} \leq c_{2}^{o}$ and we distinguish between five sub-cases defined depending on the position of $c$ relatively to $c_{1}^{e}, c_{1}^{o}, c_{2}^{e}$ and $c_{2}^{o}$. Indeed the expression of the derivative is different according to the value of $c$.

(i) If $c \leq c_{1}^{e}$ then $P^{e}=P^{o}=1$ and $\partial \bar{D} / \partial c=0$.

(ii) If $c \in\left[c_{1}^{e} ; c_{2}^{e}\right]$ then $P^{o}=1$ and $\partial \bar{D} / \partial c \geq 0$.

(iii) If $c \in\left[c_{2}^{e} ; c_{1}^{o}\right]$ then $P^{o}=1, P^{e}=\frac{1}{2}$ and $\partial \bar{D} / \partial c=0$.

(iv) If $c \in\left[c_{1}^{o} ; c_{2}^{o}\right]$ then $P^{e}=\frac{1}{2}$ and $\partial \bar{D} / \partial c \leq 0$.

(v) If $c \geq c_{2}^{o}$ then $P^{e}=P^{o}=\frac{1}{2}$ and $\partial \bar{D} / \partial c=0$.

Critical values of $\bar{D}(c)$ follow:

$$
\begin{aligned}
& \bar{D}\left(c_{1}^{e}\right)=\frac{1}{2}, \\
& \bar{D}\left(c_{2}^{e}\right)=\frac{1}{2}+\frac{(\eta+\gamma) x}{8 \Delta(\check{\alpha}+d)}, \\
& \bar{D}\left(c_{2}^{o}\right)=\frac{1}{2}-\frac{3(\eta+\gamma) x}{8 \Delta(\check{\alpha}+d)},
\end{aligned}
$$

where $\bar{\Delta}=\Delta\left(\alpha_{\text {taxi }}+d\right)$.

The variations of the difference between optimal demand for the bus and equilibrium demand for the bus are described in Table F.3. 


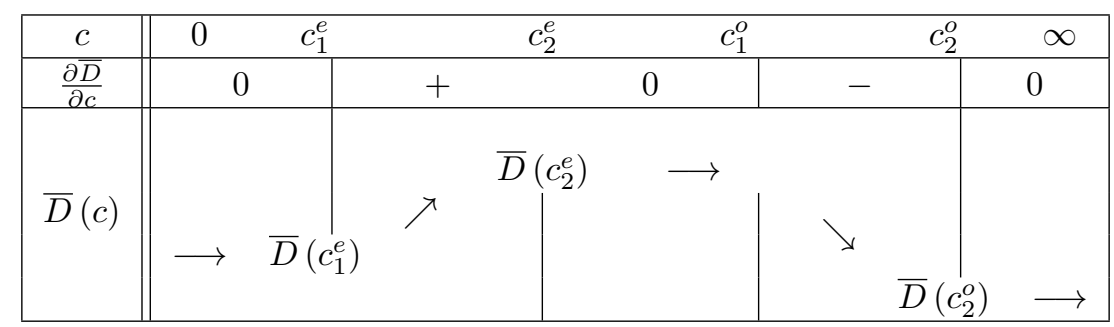

Table F.3: Variation table of the difference between optimal demand for the bus and equilibrium demand for the bus depending on the cost of reliability.

We know that $\bar{D}\left(c_{1}^{e}\right) \geq 0$ and $\bar{D}\left(c_{1}^{e}\right) \geq \bar{D}\left(c_{1}^{o}\right)$. According to Table F.3, we can distinguish between two cases where the difference between optimal and equilibrium demand for the bus is positive. First, if the minimum value of the difference, $\bar{D}\left(c_{2}^{o}\right)$ is positive, the difference is positive. Second, if this minimum value of the difference is negative, then as $\bar{D}\left(c_{2}^{e}\right) \geq 0$ and $\bar{D}(c)$ strictly decreases between $c_{1}^{o}$ and $c_{2}^{o}$, there exists a unique value of $c$ denoted $c^{c}$ for which $D_{b u s}^{o} \underset{c=c^{c}}{=} D_{b u s}^{e}$. The difference is then positive if $c \leq c^{c}$.

One critical value of the taxi operating cost $d_{1}^{c}$ may be defined such that

$$
\begin{aligned}
\bar{D}\left(c_{2}^{o}\right) \geq 0 & \Longleftrightarrow 2 \Delta(\check{\alpha}+d)-\left(3+P^{e}-4 P^{o}\right)(\eta+\gamma) x \geq 0 \\
& \Longleftrightarrow d \geq \frac{3(\eta+\gamma) x}{4 \Delta}-\check{\alpha} \equiv d_{1}^{c}
\end{aligned}
$$

We can now write

$$
\bar{D} \begin{cases}\geq 0 & \text { if } \quad d \geq d_{1}^{c}, \\ <0 & \text { or if } \quad d<d_{1}^{c} \quad \text { if } \quad d<d_{1}^{c} \quad \text { and } c>c^{c}\end{cases}
$$

\section{AppendixG. Proof of lemma 6}

The idea of the proof is that the difference between optimal demand for the bus and equilibrium demand for the bus is a function of the cost of the bus reliability $c$ and the operating cost of taxi $d$. We deal with the case where $\theta=0$. Let us recall expressions of the demand function:

$$
D_{b u s}=(1-P) \int_{\delta_{T, L}^{B}}^{\delta_{L, O}^{B}} f(\delta) d \delta+\int_{\delta_{L, O}^{B}}^{\Delta} f(\delta) d \delta,
$$

where $\delta_{L, O}^{B}=\left[\kappa+\left(\frac{1-P}{P} \eta+\beta\right) x\right] /(\check{\alpha}+\tau)$ and $\delta_{T, L}^{B}=\kappa /(\check{\alpha}+\tau)$. We therefore have:

$$
\bar{D} \equiv D_{\text {bus }}^{o}-D_{\text {bus }}^{e}=\frac{2 \Delta(\check{\alpha}+d)+\left(4 P_{\theta=0}^{o}-P_{\theta=0}^{e}\right)(\eta-\beta) x-3 \eta x}{4 \Delta(\check{\alpha}+d)}
$$

Then

$$
\bar{D} \geq 0 \Longleftrightarrow d \geq \frac{3 \eta x-\left(4 P_{\theta=0}^{o}-P_{\theta=0}^{e}\right)(\eta-\beta) x}{2 \Delta}-\check{\alpha}
$$


Considering $\max \left(4 P_{\theta=0}^{o}-P_{\theta=0}^{e}\right)=\frac{7}{2}$ and $\min \left(4 P_{\theta=0}^{o}-P_{\theta=0}^{e}\right)=1$, we can define $d_{2}^{c}$ and $d_{3}^{c}$ such as if $d \leq d_{2}^{c}$ then $\bar{D} \leq 0$ and if $d \geq d_{3}^{c}$ then $\bar{D} \geq 0$. Consequently we have $d_{2}^{c}=-\left(\frac{1}{2} \eta-\frac{7}{2} \beta\right) x / 2 \Delta-\check{\alpha}$ and $d_{3}^{c}=(2 \eta+\beta) x / 2 \Delta-\check{\alpha}$. We may write:

$$
\bar{D}\left\{\begin{array}{l}
\geq 0 \text { if } d \geq d_{3}^{c} \\
\leq 0 \text { if } d \leq d_{2}^{c}
\end{array}\right.
$$

\section{AppendixH. Discussion of Conjecture 2}

With values specified in Table 1, we can draw the curve of the difference between optimal demand for the bus and equilibrium demand for the bus depending on the operating taxi cost $d$ in Figure H.12. When $c$ is small, $P^{o}=P^{e}=1$ and when $c$ is large, $P^{o}=P^{e}=1 / 2$. The conjecture 2 is illustrated. $\bar{D}$ functions are first negative then positive. Moreover they increase with $d$. The sign of $\bar{D}$ between $d_{2}^{c}$ and $d_{3}^{c}$ depends on the values of $P^{o}$ and $P^{e}$ which depend on $c$ (see Equations (7) and (14)). Therefore we conjecture that between $d_{2}^{c}$ and $d_{3}^{c}, \bar{D}$ is positive when $c \leq c_{2}^{c}$ and negative when $c>c_{2}^{c}$, where $c_{2}^{c}$ is defined as the unique solution of $D_{\theta=0}^{o}=D_{\theta=0}^{e}$.

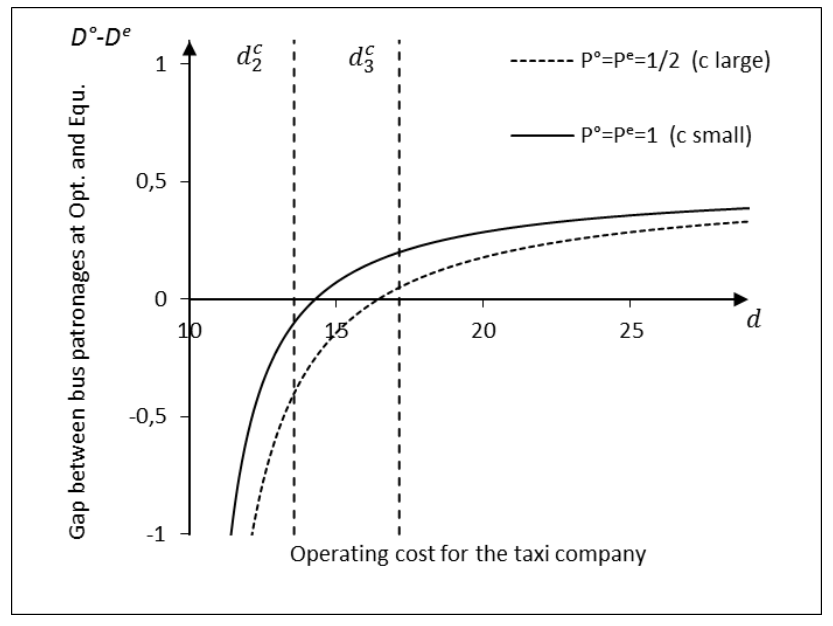

Figure H.12: Difference between optimal demand for the bus and equilibrium demand for the bus depending on operating taxi cost $d$ for Group B.

\section{AppendixI. Proof of proposition 7}

We wish to compare expected costs of Strategys $A, B$ and $O$, denoted $E C(O), E C(L)$ and $E C(T)$ respectively, to define a choice rule for a commuter in Group A. From equations (1) and (2), we can write:

$$
\begin{aligned}
& E C(O)=\kappa+(1-P)(\eta+\gamma) x, \\
& E C(L)=\kappa+(1-P) \gamma x+P(\gamma h+\eta x), \\
& E C(T)=\delta(\check{\alpha}+\tau)
\end{aligned}
$$


Therefore we have

$$
E C(O) \leq E C(L) \text { when } \frac{\eta x}{\gamma h+2 \eta x} \leq P .
$$

Using A. and A., we know that $\frac{\eta x}{\gamma h+2 \eta x} \leq \frac{1}{2}$ and that $\frac{1}{2} \leq P$. Strategy $L$ is also still dominated by Strategy $O$ and never chosen by commuter in Group $A$.

$$
E C(T)<E C(O) \text { iff } \delta<\frac{\kappa+(1-P)(\eta+\gamma) x}{(\check{\alpha}+\tau)} \equiv \delta_{T, O}^{A}
$$

\section{AppendixJ. Proof of Proposition 8}

We wish to compare expected costs of Strategys $A, B$ and $O$, denoted $E C(O), E C(L)$ and $E C(T)$ respectively, to define a choice rule for a commuter in Group B. From equations (1) and (2), we can write::

$$
\begin{aligned}
& E C(O)=\kappa+[(1-P) \eta+P \beta] x, \\
& E C(L)=\kappa+P(\gamma+\eta)(h-x), \\
& E C(T)=\delta(\check{\alpha}+\tau)
\end{aligned}
$$

Therefore we have

$$
\begin{gathered}
E C(O) \leq E C(L) \text { iff } h \geq\left(1+\frac{\frac{1-P}{P} \eta+\beta}{\eta+\gamma}\right) x \equiv h^{c}, \\
E C(T)<E C(L) \text { iff } \delta<\frac{\kappa+P(\gamma+\eta)(h-x)}{(\check{\alpha}+\tau)} \equiv \delta_{T, L^{\prime}}^{B}, \\
E C(T)<E C(O) \text { iff } \delta<\frac{\kappa+[(1-P) \eta+P \beta] x}{\check{\alpha}+\tau} \equiv \delta_{T, O}^{B} .
\end{gathered}
$$

We do not need to $\operatorname{rank} \overline{\delta_{T, L}^{B}}$ and $\delta_{T, O}^{B}$ because Strategy $O$ and Strategy $T$ dominate each other depending on $h$.

\section{AppendixK. Extension: bus and taxi fare at equilibrium}

We first solve the case where $h$ is higher than $h^{c}$ and then the case where it is lower.

When $h$ is higher than $h^{c}$, all bus users arrive at the bus stop at time $T$. The solutions of the first-order problem follow

$$
\begin{aligned}
& \kappa^{e}= \frac{1}{2}\left(\check{\triangle}^{e}-\Gamma^{e} x\right), \\
& P^{e}= \begin{cases}\frac{1}{2} & \text { if } c>c_{2}^{e}, \\
\frac{\kappa^{e} \check{\eta} x}{c \triangle^{e}} & \text { if } c \in\left[c_{1}^{e} ; c_{2}^{e}\right], \\
1 & \text { if } c<c_{1}^{e},\end{cases}
\end{aligned}
$$


where $\check{\Delta}^{e}=\Delta\left(\check{\alpha}+\tau^{e}\right), \Gamma^{e}=\left(1-P^{e}\right) \eta+(1-\theta) P^{e} \beta+\theta\left(1-P^{e}\right) \gamma, \check{\eta}=\eta-(1-\theta) \beta+\theta \gamma, c_{1}^{e} \equiv$ $\kappa^{e} \check{\eta} x / \breve{\Delta}^{e}$ and where $c_{2}^{e} \equiv 2 c_{1}^{e}$.

When $h$ is lower than $h^{c}$, then users from Group $B$ arrive late at the bus stop whereas users from Group A still arrive on time. The first-order conditions gives

$$
\begin{aligned}
& \kappa^{e}= \frac{1}{2}\left\{\check{\Delta}^{e}-(\eta+\gamma)\left[\theta\left(1-P^{e}\right) x+(1-\theta) P^{e}(h-x)\right]\right\} \\
& P^{e}= \begin{cases}\frac{1}{2} & \text { if } c>\overline{c_{2}^{e}}, \\
\frac{\kappa^{e} \eta_{h}}{c \bar{\Delta}^{e}} & \text { if } c \in\left[\overline{c_{1}^{e}} ; \overline{c_{2}^{e}}\right] \\
1 & \text { if } c<\overline{c_{1}^{e}},\end{cases}
\end{aligned}
$$

where $\check{\Delta}^{e}=\Delta\left(\check{\alpha}+\tau^{e}\right), \eta_{h} \equiv(\eta+\gamma)[x-(1-\theta) h], \overline{c_{1}^{e}} \equiv \kappa^{e} \eta_{h} / \check{\Delta}^{e}$ and where $\overline{c_{2}^{e}} \equiv 2 \overline{c_{1}^{e}}$.

\section{AppendixL. Extension: bus and taxi fare at optimum}

When $h$ is higher than $h^{c}$, the expression of the optimal level of reliability is

$$
P^{o}= \begin{cases}\frac{1}{2} & \text { if } c>\overline{c_{4}^{o}}, \\ \frac{\check{\eta} x \check{\Delta}^{o}-\Theta+(1-\theta)(\eta-\beta) \beta x^{2}}{c \check{\Delta}^{o}-\Theta} & \text { if } c \in\left[\bar{c} ; \overline{c_{4}^{o}}\right], \\ 1 & \text { if } c<\overline{c_{3}^{o}},\end{cases}
$$

where $\check{\eta}=\eta-(1-\theta) \beta+\theta \gamma, \check{\Delta}^{o}=\Delta\left(\check{\alpha}+\tau^{o}\right)$,

$\Theta \equiv \theta[(\eta+\gamma) x]^{2}+(1-\theta)[(\eta-\beta) x]^{2}, \overline{c_{3}^{o}} \equiv\left[\check{\Delta}^{o} \check{\eta} x+(1-\theta)(\eta+\beta) \beta x^{2}\right] / \check{\Delta}^{o}$ and where $\overline{c_{4}^{o}} \equiv\left[2 \check{\Delta}^{o} \check{\eta} x-\theta((\eta+\gamma) x)^{2}-(1-\theta)\left(\eta^{2}-\beta^{2}\right) x^{2}\right] / \check{\Delta}^{o}$.

When $h$ is lower than $h^{c}$, the solution of the optimization problem is

$$
P^{o}= \begin{cases}\frac{1}{2} & \text { if } c>\overline{c_{2}^{o}}, \\ \overline{c \breve{\Delta}^{o}-(\eta+\gamma)^{2}\left[(1-\theta)(h-x)^{2}+\theta x^{2}\right]} & \text { if } c \in\left[\overline{c_{1}^{o}} ; \overline{c_{2}^{o}}\right], \\ 1 & \text { if } c<\overline{c_{1}^{o}},\end{cases}
$$

where $\eta_{h} \equiv(\eta+\gamma)[x-(1-\theta) h], \check{\Delta}^{o}=\Delta\left(\check{\alpha}+\tau^{o}\right)$,

$\overline{c_{1}^{o}} \equiv\left[\check{\Delta}^{o} \eta_{h}+(\eta+\gamma)^{2}(1-\theta)(h-x)^{2}\right] / \check{\Delta}^{o}$

and where $\overline{c_{2}^{o}} \equiv\left\{2 \check{\Delta}^{o} \eta_{h}-(\eta+\gamma)^{2}\left[\theta x^{2}-(1-\theta)(h-x)^{2}\right]\right\} / \check{\Delta}^{o}$.

\section{AppendixM. Proof of Proposition 9}

We wish to show that $P^{e}$, the probability of the bus being on time at equilibrium, and $\kappa^{e}$, the bus fare, increase with $\tau$ the taxi fare. We already have proved it is true when $h^{c} \leq h$ (see Appendix). We now need to prove it when $h^{c}>h$. As before, we first show that $\partial P^{e} / \partial \tau \geq 0$ (i) and that $\partial \kappa^{e} / \partial \tau \geq 0$ (ii). Then we check that boundaries of interval, $c_{1}^{e} \prime$ (iii) and $c_{2}^{e}$ (iv), increase with $\tau$. Let us recall 
expressions of equilibrium variables (see equations (6) and (7)):

$$
\begin{gathered}
\kappa^{e}=\frac{1}{2}\left[\check{\Delta}^{e}-(\eta+\gamma)\left[\theta\left(1-P^{e}\right) x+(1-\theta) P^{e}(h-x)\right]\right] \\
P^{e}= \begin{cases}\frac{1}{2} & \text { if } c>\overline{c_{2}^{e}}, \\
\frac{\kappa^{e} \eta_{h}}{c \bar{\Delta}^{e}} & \text { if } c \in\left[\overline{c_{1}^{e}} ; \overline{c_{2}^{e}}\right], \\
1 & \text { if } c<\overline{c_{1}^{e}},\end{cases}
\end{gathered}
$$

where $\check{\Delta}^{e}=\Delta\left(\check{\alpha}+\tau^{e}\right), \eta_{h} \equiv(\eta+\gamma)[x-(1-\theta) h], c_{1}^{e} \prime \equiv \kappa^{e} \eta_{h} / \check{\Delta}^{e}$ and where $\overline{c_{2}^{e}} \equiv 2 \overline{c_{1}^{e}}$. By substituting $\kappa^{e}$ in $P^{e}$, we obtain

$$
P^{e}= \begin{cases}\frac{1}{2} & \text { if } c>c_{2}^{e} \\ \frac{\left[\check{\Delta}^{e}-(\eta+\gamma) \theta x\right] \eta_{h}}{2 c \check{\Delta}^{e}-\eta_{h}^{2}} & \text { if } c \in\left[c_{1}^{e} / c_{2}^{e}\right] \\ 1 & \text { if } c<c_{1}^{e},\end{cases}
$$

where $\check{\Delta}^{e}=\Delta\left(\check{\alpha}+\tau^{e}\right), \eta_{h} \equiv(\eta+\gamma)[x-(1-\theta) h]$,

$c_{1}^{e} \boldsymbol{I} \equiv\left[\check{\Delta}^{e}-(\eta+\gamma)(1-\theta)(h-x)\right] \eta_{h} / 2 \check{\Delta}^{e}$ and where

$c_{2}^{e} \prime \equiv\left[2 \check{\Delta}^{e}-(\eta+\gamma)((2 \theta-1) x+(1-\theta) h)\right] \eta_{h} / 2 \check{\Delta}^{e}$. We now derive $P^{e}, \kappa^{e}, c_{1}^{e}$ and $c_{2}^{e}$ on $\tau^{e}$.

(i) $\frac{\partial P^{e}}{\partial \tau^{e}}=\Delta(\eta+\gamma) \eta_{h} \frac{2 c \theta x-\eta_{h}[x-(1-\theta) h]}{\left[2 c \check{\Delta}^{e}-\eta_{h}^{2}\right]^{2}}$ so $\frac{\partial P^{e}}{\partial \tau} \geq 0$ if $c \geq \frac{\eta_{h}[x-(1-\theta) h]}{2 \theta x}$. Let us substitute $c$ by $c_{1}^{e}$ ' the minimal value of the interval $\left[c_{1}^{e} / ; c_{2}^{e}\right]$. Thus

$$
c_{1}^{e} \prime-\frac{\eta_{h}[x-(1-\theta) h]}{2 \theta x}=\frac{\left[\check{\Delta}^{e}-\theta x(\eta+\gamma)\right](1-\theta)(h-x) \eta_{h}}{2 \theta x \check{\Delta}^{e}}
$$

Yet $\frac{\left[\check{\Delta}^{e}-\theta x(\eta+\gamma)\right](1-\theta)(h-x) \eta_{h}}{2 \theta x \check{\Delta}^{e}} \geq 0$. We therefore have $\frac{\partial P^{e}}{\partial \tau} \geq 0$;

(ii) $\frac{\partial \kappa^{e}}{\partial \tau^{e}}=\frac{\Delta}{2}+\frac{\partial P^{e}}{\partial \tau^{e}} \frac{\eta_{h}}{2} \geq 0$ by A.2,

(iii) $\frac{\partial c_{1}^{e}}{\partial \tau^{e}}=\frac{(\eta+\gamma)^{2}(1-\theta) \eta}{2 \Delta\left(\tilde{\alpha}+\tau^{e}\right)^{2}} \geq 0$,

(iv) $\frac{\partial c_{2}^{e}}{\partial \tau^{e}}=\frac{(\eta+\gamma)((2 \theta-1) x+(1-\theta) h) \eta_{h}}{2 \Delta\left(\check{\alpha}+\tau^{e}\right)^{2}} \geq 0$.

$P^{e}$, the probability of the bus being on time at equilibrium, and $\kappa^{e}$, the bus fare, increase well with $\tau^{e}$ the taxi fare.

\section{AppendixN. Proof of Proposition 10}

We wish to show that the probability of the bus being on time is higher in the optimal situation than in equilibrium when $c$ is higher than a critical value $c^{c}$. For that, we need to show that the sign of the result of $P_{\theta=1}^{o}-P_{\theta=1}^{e}$ depends on $c$. 
If $h>h^{c}$,

$$
\begin{aligned}
P^{o}-P^{e}= & \frac{c \check{\Delta}^{o}\left\{2 \check{\eta} x \check{\Delta}^{o}-4\left[\Theta-(1-\theta)(\eta-\beta) \beta x^{2}\right]+(\eta+\theta \gamma) \check{\eta} x^{2}\right\}}{\left(c \check{\Delta}^{o}-\Theta\right)\left[2 c \check{\triangle}^{e}-(\check{\eta} x)^{2}\right]} \\
& -\frac{\check{\Delta}^{o}(\check{\eta} x)^{3}+\check{\eta} x \Theta\left[2 \check{\triangle}^{o}-(1-\theta) \beta x\right]}{\left(c \check{\Delta}^{o}-\Theta\right)\left[2 c \check{\triangle}^{e}-(\check{\eta} x)^{2}\right]}
\end{aligned}
$$

and if $h \leq h^{c}$,

$$
\begin{aligned}
P^{o}-P^{e}= & \frac{c \check{\Delta}^{o}\left[2 \eta_{h} \check{\Delta}^{o}-(\eta+\gamma) \theta x\left[4(\eta+\gamma) x+\eta_{h}\right]\right]}{\left\{c \check{\Delta}^{o}-(\eta+\gamma)^{2}\left[(1-\theta)(h-x)^{2}+\theta x^{2}\right]\right\}\left\{4 c \check{\Delta}^{o}-\eta_{h}^{2}\right\}} \\
& -\frac{\check{\Delta}^{o} \eta_{h}^{3}+}{\left\{c \check{\Delta}^{o}-(\eta+\gamma)^{2}\left[(1-\theta)(h-x)^{2}+\theta x^{2}\right]\right\}\left\{4 c \check{\Delta}^{o}-\eta_{h}^{2}\right\}} \\
& +\frac{\eta_{h}(\eta+\gamma)^{2}\left\{\left[2 \check{\Delta}^{o}-(\eta+\gamma) \theta x\right]\left[(1-\theta)(h-x)^{2}+\theta x^{2}\right]+\eta_{h} \theta x^{2}\right\}}{\left\{c \check{\Delta}^{o}-(\eta+\gamma)^{2}\left[(1-\theta)(h-x)^{2}+\theta x^{2}\right]\right\}\left\{4 c \check{\Delta}^{o}-\eta_{h}^{2}\right\}}
\end{aligned}
$$

We also can define $c^{c}$ such as if $c>c^{c}$ then $P^{o} \geq P^{e}$ and if $c \leq c^{c}$ then $P^{o} \leq P^{e}$ :

$$
c^{c}=\left\{\begin{array}{ll}
\frac{\check{\Delta}^{o}(\check{\eta} x)^{3}-\check{\eta} x \Theta\left(2 \check{\Delta}^{o}-(1-\theta) \beta x\right)}{\check{\Delta}^{o}\left\{2 \check{\eta} x \check{\Delta}^{o}-4\left[\Theta-(1-\theta)(\eta-\beta) \beta x^{2}\right]+(\eta+\theta \gamma) \tilde{\eta} x^{2}\right\}} & \text { if } h<h^{c} \\
\frac{\check{\Delta}^{o} \eta_{h}^{3}-\eta_{h}(\eta+\gamma)^{2}\left\{\left[2 \check{\Delta}^{o}-(\eta+\gamma) \theta x\right]\left[(1-\theta)(h-x)^{2}+\theta x^{2}\right]+\eta_{h} \theta x^{2}\right\}}{\check{\Delta}^{o}\left[2 \eta_{h} \check{\Delta}^{o}-4(\eta+\gamma)^{2} \theta x^{2}+(\eta+\gamma) \theta x \eta_{h}\right]} & \text { if } h \geq h^{c}
\end{array},\right.
$$

where $\check{\eta}=\eta-(1-\theta) \beta+\theta \gamma, \check{\Delta}^{o}=\Delta\left(\check{\alpha}+\tau^{o}\right), \Theta \equiv \theta[(\eta+\gamma) x]^{2}+(1-\theta)[(\eta-\beta) x]^{2}$ and $\eta_{h} \equiv$ $(\eta+\gamma)[x-(1-\theta) h]$. 